\title{
Pacific
}

Journal of

Mathematics

\section{SOME REPRESENTATIONS OF TAF ALGEBRAS}

\author{
JOHN LINDSAY ORR AND JUSTIN PETERS
}

Volume $167 \quad$ No. 1 


\title{
SOME REPRESENTATIONS OF TAF ALGEBRAS
}

\author{
John L. OrR And Justin R. Peters
}

\begin{abstract}
The study of triangular AF algebras has focused mostly on the classification, and although some canonical representations of the important examples have long been known, there has been little systematic study of the reprresentation theory of these algebras. The representations considered here are those which are restrictions of starrepresentations of the full AF algebra. Furthermore, attention will mostly be restricted to representations which map the masa of the triangular AF algebra weakly densely into a masa of $B(H)$. Such representations have a convenient description using groupoids. Much of the paper consists of examples illustrating what can and cannot occur.
\end{abstract}

0. Introduction. Thirty years ago Kadison and Singer began the study of triangular operator algebras in Hilbert space; a subalgebra $\mathcal{S}$ of the bounded operators on a Hilbert space $\mathcal{H}$ was called triangular in case $\mathcal{S} \cap \mathcal{S}^{*}$ is a maximal abelian subalgebra (masa) of $\mathcal{B}(\mathcal{H}) ; \mathcal{S}^{*}$ is the set of adjoints of elements of $\mathcal{S}$. Since their paper [5], a large body of work concerning triangular subalgebras and nest subalgebras in Hilbert space has emerged (cf. [13], [3]). Quite recently there has been an interest in triangular subalgebras of AF $C^{*}$-algebras (cf. [8], [4], [9], [10], [15], [16], to name a few). While analogues between the two theories have been noticed - indeed, they are hard to ignore - no direct connection has been established between them. If $\mathfrak{A}$ is $A F$, a norm-closed subalgebra $\mathcal{T} \subset \mathfrak{A}$ is said to be a triangular $\mathrm{AF}$ (TAF) if $\mathcal{T} \cap \mathcal{T}^{*}$ is a certain kind of masa in $\mathfrak{A}$ (see Sec. II), and it is strongly maximal triangular if in addition $\mathcal{T}+\mathcal{T}^{*}$ is dense in $\mathfrak{A}$. The natural connection between strongly maximal TAF algebras and nest algebras is established by observing that if $\rho$ is a representation of the $\operatorname{AF} C^{*}$-algebra $\mathfrak{A}$ such that $\rho(\mathfrak{A})$ is 
weakly dense in $\mathcal{B}\left(\mathcal{H}_{\rho}\right)$, and $\mathcal{T}$ is a strongly maximal triangular subalgebra of $\mathfrak{A}$ such that $\rho \overline{(\mathcal{T})}^{\text {wk }}$ contains a masa, then $\rho(\mathcal{T})$ is weakly dense in a nest algebra in $\mathcal{B}\left(\mathcal{H}_{\rho}\right)$ (Proposition 0.1 ). A tractible class of such representations are those which map the diagonal of $\mathcal{T}$ to a dense subset of a masa in $\mathcal{B}\left(\mathcal{H}_{\rho}\right)$. We shall call such representations masa-preserving and will characterize them up to unitary equivalence in Section II. Indeed, in Theorem II.1 we show that any such representation is unitarily equivalent to one constructed from a certain quasi-invariant measure and a 1-cocycle. Special cases have been considered by Stratila and Voiculescu ([14, p. 51]). If $\rho$ is such a representation, and $\tilde{\mathcal{T}}=\rho \overline{(\mathcal{T})}^{\text {wk }}$ (weak closure), some obvious questions present themselves: is $\tilde{\mathcal{T}}$ a triangular subalgebra of $\mathcal{B}\left(\mathcal{H}_{\rho}\right)$ ? If $\mathcal{T}$ is a nest TAF-subalgebra of $\mathfrak{A}$, is $\tilde{\mathcal{T}}$ a triangular nest subalgebra of $\mathcal{B}\left(\mathcal{H}_{\rho}\right)$ ? The answers to these questions are in general both no. As to the first question, far from being triangular, it can happen that $\tilde{\mathcal{T}}=\mathcal{B}\left(\mathcal{H}_{\rho}\right)$ (Example I.3). As to the second, Example IV.8 shows there is a triangular nest subalgebra $\mathcal{T}$ of a UHF algebra $\mathfrak{A}$ such that $\tilde{\mathcal{T}}$, while necessarily a nest algebra, fails to be triangular in $\mathcal{B}\left(\mathcal{H}_{\rho}\right)$. Thus in general, the map $\mathcal{T} \rightarrow \tilde{\mathcal{T}}$ does not preserve triangularity.

D. Larson posed the question as to whether if $\mathcal{T}$ is a strongly maximal TAF subalgebra of a UHF algebra $\mathfrak{A}$, there is a faithful representation $\rho$ of $\mathfrak{A}$ such that the weak closure of $\rho(\mathcal{T})$ is a triangular nest algebra in $\mathcal{B}\left(\mathcal{H}_{\rho}\right)$. Proposition II.3 provides a positive answer.

A more delicate question is this: given a TAF algebra $\mathcal{T}$ and a representation $\rho$ such that $\rho(\mathcal{T})$ is weakly dense in the nest algebra $\operatorname{Alg}(\mathcal{N})$, what can be said about the order type of $\mathcal{N}$ ? While it is easily seen that the refinement algebra (Example I.1) cannot be weakly densely represented in $\operatorname{Alg} \mathcal{N}$ if $\mathcal{N}$ has the order type of a subset of the integers, it is less obvious but nonetheless true that the standard embedding algebra (Example I.2) cannot be weakly densely embedded in the Volterra nest algebra. Moreover, if the standard embedding algebra is weakly densely embedded in $\operatorname{Alg} \mathcal{N}$, then $\mathcal{N}$ has the order type of a subset of the integers. Furthermore, if we restrict to masa-preserving representations, then the same conclusion holds for the class of $\mathbb{Z}$-analytic TAF algebras. However, there are TAF algebras which can be weakly densely embedded both 
in the Volterra nest algebra, and in $\operatorname{Alg} \mathcal{N}$, where $\mathcal{N}$ has the order type of the natural numbers. Section IV entails examples of representations with multiplicity. In IV.3, the refinement algebra is weakly embedded in $\operatorname{Alg} \mathcal{N}$ where $\mathcal{N}$ is a nest of multiplicity two. This is by a finite group construction.

The examples of this paper indicate there is a richness in the behavior of representations of TAF algebras which might not have been expected. Perhaps in the future there will be a general solution to the problem posed in the paragraph above.

The paper is organized as follows:

0 . Introduction

I. Review of important examples and their natural representations

II. Masa-preserving representations

II.1 The general construction: the measure-cocycle characterization

II.2 Properties of masa-preserving representations

II.3 Examples of measure-cocycle constructions

III. Attainable order types

III.1 Algebras in which Lat $\mathcal{T}$ generates the diagonal

III.2 Representations of the standard algebra

III.3 Masa-preserving representations of $\mathbb{Z}$-analytic algebras

III.4 On the variety of attainable order types

IV. Multiplicity of represented algebras

IV.1 A masa-preserving representation with a multiplicity two nest

IV.2 The failure of representations to preserve triangularity for nest algebras

IV.3 A non masa-preserving representation of the refinement algebra with a multiplicity two nest

The main idea for the following is due to Arveson.

Proposition 0.1. Let $\mathcal{T}$ be a strongly maximal triangular subalgebra of the $A F C^{*}$-algebra $\mathfrak{A}$. If $\rho$ is a representation of $\mathfrak{A}$ which is weakly dense in $\mathcal{B}\left(\mathcal{H}_{\rho}\right)$ and if the weak closure of $\rho(\mathcal{T})$ contains 
a masa, then $\rho(\mathcal{T})$ is weakly dense in a nest algebra.

Proof. Let $\mathcal{M}$ be a masa contained in $\rho \overline{(\mathcal{T})}^{\text {wk }}$ and $P_{1}, P_{2}$ be two two invariant projections of $\rho(\mathcal{T})$ which are incomparable. Then both $P_{1}, P_{2}$ belong to $\mathcal{M}$, and $E_{1}=P_{1} P_{2}^{\perp}, E_{2}=P_{2} P_{1}^{\perp}$ satisfy $E_{1} \rho(\mathcal{T}) E_{2}=0$ and $E_{2} \rho(\mathcal{T}) E_{1}=0$. Now of course $E_{1}\left(\rho(\mathcal{T})+\rho(\mathcal{T})^{*}\right)$ $E_{2} \neq 0$, so that $E_{1} \rho(\mathcal{T})^{*} E_{2} \neq 0$. But this is $\left(E_{2} \rho(\mathcal{T}) E_{1}\right)^{*}$, which is zero.

Since Lat $\rho(\mathcal{T})$ is a nest, the conclusion follows from [3, Corollary 15.12].

\section{Review of important examples and their natural rep- resentations.}

EXAMPLE I.1. Let $\mathcal{T}_{n}$ be the upper triangular matrices in $M_{2^{n}}$, and let $\mathcal{T}=\underline{\lim }\left(\mathcal{T}_{n}, \nu_{n}\right)$ be the canonical nest TUHF algebra considered in $[8,1.1]$. It has been observed that $\mathcal{T}$ can be represented as a weakly dense subalgebra of the Volterra nest algebra. Let $\left\{e_{i j}^{(n)}\right\}_{1 \leq i, j \leq 2^{n}}$ be a system of matrix units for $\mathfrak{A}_{n}=M_{2^{n}}$, and let $\rho$ be the representation of $\mathfrak{A}=\underline{\varliminf}\left(\mathfrak{A}_{n}, \nu_{n}\right)$ on $\mathcal{H}=L^{2}([0,1], m)(m$ Lebesgue measure) given by

$$
\left(\rho\left(e_{i j}^{(n)}\right) f\right)(t)=\chi_{\left[\frac{i-1}{2^{n}}, \frac{i}{2^{n}}\right]}(t) f\left(t+\frac{j-i}{2^{n}}\right) .
$$

$\rho$ extends by linearity to a representation of $\mathfrak{A}_{n}$. Note that

$$
\rho\left(e_{i j}^{(n)}\right)=\rho\left(e_{2 i-12 j-1}^{(n+1)}\right)+\rho\left(e_{2 i 2 j}^{(n+1)}\right),
$$

so that $\rho\left(\mathfrak{A}_{n}\right)=\rho\left(\nu_{n}\left(\mathfrak{A}_{n}\right)\right)$, and hence $\rho$ extends to a representation of $\mathfrak{A}$. Since $\mathfrak{A}=\operatorname{UHF}\left(2^{\infty}\right)$ is simple, $\rho$ is necessarily faithful, and as we will see later, an irreducible representation of $\mathfrak{A}$. Now if $f \in \mathcal{H}$ is supported on $\left[0, t_{0}\right]\left(0<t_{0}<1\right)$ and $i \leq j$, then $\rho\left(e_{i j}^{(n)}\right) f$ is supported on $\left[0, t_{0}-\frac{j-i}{2^{n}}\right] \subseteq\left[0, t_{0}\right]$. As $\mathcal{T}$ is the closed linear span of the matrix units $\left\{e_{i j}^{(n)}: 1 \leq i \leq j \leq 2^{n} n=1,2, \ldots\right\}, \rho(\mathcal{T})$ leaves the nest $\mathcal{N}=\left\{\chi_{\left[0, t_{0}\right]}: 0<t_{0}<1\right\}$ invariant. Let $\tilde{\mathcal{T}}=\overline{\rho(\mathcal{T})}^{\text {wk }}$; since $\rho(\mathcal{T})$ contains all dyadic left translations. $\tilde{\mathcal{T}}$ contains all left translations. Since $\rho(\mathcal{T})$ contains all multiplication operators of the 
form $\sum_{i=1}^{2^{n}} a_{\imath} P_{\left[\frac{2-1}{2^{n}}, \frac{\imath}{2^{n}}\right]}\left(P_{I}\right.$ the projection onto the subspace of functions supported on $I), \rho(\mathcal{T})$ also contains all multiplication operators by continuous functions, so $\tilde{\mathcal{T}}$ contains the masa of multiplication operations by $L^{\infty}$-functions. We conclude that Lat $\tilde{\mathcal{T}}=\mathcal{N}$. It follows from Proposition 0.1 that $\tilde{\mathcal{T}}=\operatorname{Alg} \mathcal{N}$. Thus $\tilde{\mathcal{T}}$ is the Volterra nest algebra.

EXAMPLE I.2. Let $\mathcal{T}=\underline{\varliminf}\left(\mathcal{T}_{n}, \sigma_{n}\right)$ be the standard embedding TUHF algebra considered in $[\mathbf{2}],[\mathbf{8}, 1.1]\left(\mathcal{T}_{n}, \mathfrak{A}_{n}\right.$ as above). R. Smith has observed that $\mathcal{T}$ can be represented as a weakly dense subalgebra of Alg $\mathcal{N}$, where $\mathcal{N}=\{0\} \cup\{1\} \cup\left\{P_{\langle 1, \ldots, n\rangle}: n=1,2, \ldots\right\}$, $P_{\langle 1, \ldots, n\rangle}$ the projection onto the span of the basis vectors $\xi_{1}, \xi_{2}, \ldots, \xi_{n}$ in $\ell^{2}(\mathbb{N})$. Define

$$
\rho\left(e_{i \jmath}^{(n)}\right) \xi_{\ell}= \begin{cases}\xi_{i+k \cdot 2^{n}}, & \text { if } \ell=j+k \cdot 2^{n} \\ 0, & \text { otherwise }\end{cases}
$$

for any $k=0,1,2, \ldots \rho$ extends linearly to a representation of $\mathfrak{A}_{n}$ and since

$$
\rho\left(e_{i j}^{(n)}\right)=\rho\left(e_{i j}^{(n+1)}\right)+\rho\left(e_{i+2^{n}}^{(n+1)} j+2^{n}\right),
$$

$\rho\left(\mathfrak{A}_{n}\right)=\rho\left(\sigma_{n}\left(\mathfrak{A}_{n}\right)\right)$, so $\rho$ extends to a representation of $\mathfrak{A}$. This is faithful, and (as we show later) irreducible. Since $\rho\left(e_{i j}^{(m)}\right) \quad(i \leq j)$ leaves the subspaces $\left\langle\xi_{1}, \ldots, \xi_{n}\right\rangle$ invariant, so does $\rho(\mathcal{T})$. Notice that $P_{\langle 1, \ldots, n\rangle}$ is the weak limit of $\rho\left(e_{1}^{(m)}+\cdots+e_{n}^{(m)}\right)$ as $m \rightarrow \infty$. It will be shown in II.3.1 that the projections $\mathcal{N}=\left\{P_{\langle 1, \ldots, n\rangle}: n=\right.$ $1,2, \ldots\} \cup\{0,1\}$ are exactly Lat $\rho(\mathcal{T})$, and that $\rho(\mathcal{T})$ is weakly dense in $\operatorname{Alg} \mathcal{N}$.

EXAMPLE I.3. Let $\mathcal{T}$ be as in Example 2; here we represent $\mathcal{T}$ on $\mathcal{H}=L^{2}[0,1]$. If $1 \leq i \leq 2^{n}$, let $i-1=\sum_{\ell=0}^{n-1} i_{\ell} 2^{n-\ell-1}$ be its dyadic expansion. Let $\sigma_{n}(i)$ be the integer obtained by reversing the lexicographic order (changing notation from I.2): $\sigma_{n}(i)-1=$ $\sum_{\ell=0}^{n-1} i_{\ell} 2^{\ell}$. Thus $\sigma_{n}$ is a permutation of $\left\{1, \ldots, 2^{n}\right\}$, and for $1 \leq i \leq$ $2^{n}, \sigma_{n+1}(i)=2 \sigma_{n}(i)-1$, and $\sigma_{n+1}\left(i+2^{n}\right)=2 \sigma_{n}(i)$. Define the representation $\rho$ by

$$
\left(\rho\left(e_{i j}^{(n)}\right) f\right)(t)=\chi_{\left[\frac{\sigma_{n}(2)-1}{2^{n}}, \frac{\sigma_{n}(2)}{2^{n}}\right]}(t) f\left(t+\frac{\sigma_{n}(j)-\sigma_{n}(i)}{2^{n}}\right) .
$$


Extend $\rho$ by linearity to a representation of $\mathfrak{A}_{n}=M_{2^{n}}$ and check that $\rho\left(e_{i j}^{(n)}\right)=\rho\left(e_{i j}^{(n+1)}\right)+\rho\left(e_{i+2^{n}}^{(n+1)} j+2^{n}\right)$, so $\rho$ is a representation of $\mathfrak{A}$. Later we will show that $\rho$ is an irreducible representation of $\mathcal{T}$ : Lat $\rho(\mathcal{T})=\{0,1\}$, and $\rho \overline{(\mathcal{T})}^{\text {wk }}=\mathcal{B}(\mathcal{H})(=\operatorname{Alg}\{0,1\})$. (See II.3.2.)

EXAMPLE I.4. Let $\mathfrak{A}_{n}=M_{4^{n}},\left\{e_{i j}^{(n)}\right\}_{1 \leq i, j \leq 4^{n}}$ a system of matrix units for $\mathfrak{A}_{n}$, and $\gamma_{n}: \mathfrak{A}_{n} \hookrightarrow \mathfrak{A}_{n+1}$ the embedding

$$
\begin{aligned}
\gamma_{n}\left(e_{i j}^{(n)}\right)= & e_{2 i-12 j-1}^{(n+1)}+e_{2 i 2 j}^{(n+1)} \\
& +e_{2 \cdot 4^{n}+2 i-12 \cdot 4^{n}+2 j-1}^{(n+1)}+e_{2 \cdot 4^{n}+2 i 2 \cdot 4^{n}+2 j}^{(n+1)} .
\end{aligned}
$$

$\gamma_{n}$ is the result of applying the standard embedding followed by the nest embedding $M_{2^{2 n}} \hookrightarrow M_{2^{2 n+1}} \hookrightarrow M_{2^{2 n+2}}$ (or in the reverse order, since the two embeddings commute). Let $\mathfrak{A}=\underline{\lim }\left(\mathfrak{A}_{n}, \gamma_{n}\right)$, $\mathcal{T}=\underline{\varliminf}\left(\mathcal{T}_{n}, \gamma_{n}\right)$, where $\mathcal{T}_{n}$ is the upper triangular matrix algebra in $\mathfrak{A}_{n} . \mathcal{T}$ has been called the alternation triangular algebra. A. Hopenwasser has observed that $\mathcal{T}$ can be represented as a $\sigma$-weakly dense subalgebra of $\operatorname{Alg} \mathcal{N}$, where $\mathcal{N}$ is the Volterra nest in $L^{2}[0, \infty)$. Note that, for $f \in L^{2}[0, \infty)$

$$
\left(\rho\left(e_{i j}^{(n)}\right) f\right)(t)=\sum_{k=0}^{\infty} \chi_{\left[\frac{i-1}{2^{n}}+k \cdot 2^{n}, \frac{i}{2^{n} n}+k \cdot 2^{n}\right]}(t) f\left(t+\frac{j-i}{2^{n}}\right)
$$

extends by linearity to a representation of $\mathfrak{A}_{n}$, and that $\rho\left(e_{i j}^{(n)}\right)=$ $\rho\left(\gamma_{n}\left(e_{i j}^{(n)}\right)\right)$, so that $\rho$ in fact gives a representation of $\mathfrak{A}$. (See II.3.2.)

\section{Masa-preserving representations.}

II.1. The General Construction: the measure-cocycle characterization. The representations in Section $I$ all satisfy $\rho{\overline{\left(\mathcal{T} \cap \mathcal{T}^{*}\right)}}^{\text {wk }}$ is a masa in $\mathcal{B}\left(\mathcal{H}_{\rho}\right)$. In this section we present a general method for constructing all such representations. This requires some notation from the theory of groupoids, so we begin with a short introduction. (For more details concerning AF algebras, groupoids, cf. $[14],[6],[8]$, or $[\mathbf{1 2}]$.)

Let $\mathfrak{A}$ be a (unital) AF algebra. A masa $\mathcal{D} \subset \mathfrak{A}$ will be called an SV masa if there is an increasing sequence $\left\{\mathfrak{A}_{n}\right\}_{n=1}^{\infty}$ of finite 
dimensional subalgebras with $\mathfrak{A}=\overline{\cup \mathfrak{A}_{n}}$ such that $\mathcal{D}_{n}=\mathcal{D} \cap \mathfrak{A}_{n}$ is a masa in $\mathfrak{A}_{n}, n=1,2, \ldots$ Then $\mathcal{D}=\overline{\cup \mathcal{D}_{n}}$. A system of matrix units can be chosen for $\mathfrak{A}_{n}(n \geq 1)$ such that each matrix unit in $\mathfrak{A}_{n}$ is a sum of matrix units in $\mathfrak{A}_{n+1}$, and each matrix unit in $\mathcal{D}_{n}$ is a sum of matrix units in $\mathcal{D}_{n+1}$. A norm-closed subalgebra $\mathcal{T} \subset \mathfrak{A}$ is TAF if $\mathcal{T} \cap \mathcal{T}^{*}$ is an SV masa in $\mathfrak{A}$. $\mathcal{T} \cap \mathcal{T}^{*}$ is called the diagonal of $\mathcal{T}$.

Let $\mathcal{D} \subset \mathfrak{A}$ be an SV masa, and $X=\mathcal{D}^{\wedge}$, the Gelfand spectrum. If $x \in X$, there is a decreasing sequence of projections $\left\{p_{n}\right\}_{n=1}^{\infty}$ with $\bigcap_{n=1}^{\infty} \hat{p}_{n}=\{x\}\left(\hat{p}_{n}\right.$ the spectrum of $p_{n}$ in $\left.X\right)$. In fact, $p_{n}$ can be chosen as a diagonal matrix unit in $\mathfrak{A}_{n}$. If $v$ is a matrix unit in $\mathfrak{A}$ with $x \in \widehat{v v^{*}}$, then there is an $n \in \mathbb{N}$ such that for $n \geq N$, $\left\{v^{*} p_{n} v\right\}_{n \geq N}$ forms a decreasing set of diagonal projections, and the intersection $\bigcap_{n=N}^{\infty} \widehat{v^{*} p_{n} v}$ is a singleton, say $y$. Write $\sigma_{v}(x)=y$ or, equivalently, $(x, y) \in \hat{v}$, the graph of $v$. In this way, $v$ is viewed as a partial homeomorphism of $X$, with domain $r(v)=\widehat{v v^{*}}$ and range $d(v)=\widehat{v^{*} v}$. Write $[x]$ to denote the orbit of $x$; i.e. $[x]=\left\{\sigma_{v}(x): v\right.$ a matrix unit of $\mathfrak{A}$ with $\left.x \in \widehat{v v^{*}}\right\}$. Each orbit is countable. If $\mathcal{T} \subset \mathfrak{A}$ is a TAF subalgebra with $\mathcal{T} \cap \mathcal{T}^{*}=\mathcal{D}$, write $x \prec y$ if $y=\sigma_{v}(x)$ for some matrix unit $v \in \mathcal{T}$. This gives a partial order on each equivalence class in $X$. This is a total order on each equivalence class iff $\mathcal{T}$ is strongly maximal (i.e., $\mathcal{T}+\mathcal{T}^{*}$ is dense in $\mathfrak{A}$ ) [15].

If $\mathcal{R}=\cup\{\hat{v}: v$ a matrix unit of $\mathfrak{A}\} \subset X \times X, \mathcal{R}$ is called the groupoid associated with the pair $(\mathfrak{A}, \mathcal{D}) . \mathcal{R}$ is topologized by letting the compact-open sets $\hat{v}$ form a base for the topology. If $\mathcal{P}=\cup\{\hat{v}$ : $v$ is a matrix unit of $\mathcal{T}\}$, then $\mathcal{P} \subset \mathcal{R}$ is called the fundamental relation of $\mathcal{T}$.

Let $\mathcal{G}$ denote the inverse semigroup of partial homeomorphisms $\sigma_{v}$ associated with the matrix units of $\bigcup_{n} \mathfrak{A}_{n}$. If $\mu$ is a measure on $X$, we will write $\mu \circ \sigma_{v}$ for the measure $\mu \circ \sigma_{v}(E)=\mu\left(\sigma_{v}(E)\right)$. A Borel probability measure $\mu$ on $X$ is $\mathcal{G}$-quasi-invariant if for each matrix unit $v$ the measures $\mu \circ \sigma_{v}, \mu$ are equivalent (as measures on $\widehat{r(v)}=\widehat{v v^{*}}$.) If $\mathcal{R}$ is the groupoid of $(\mathfrak{A}, \mathcal{D})$, a 1-cocycle on $\mathcal{R}$ is a map $\alpha: \mathcal{R} \rightarrow \mathbb{C}$ with $|\alpha|=1$ satisfying

$$
\alpha(x, y) \alpha(y, z)=\alpha(x, z)
$$


for all pairs $(x, y),(y, z) \in \mathcal{R}$.

Given a matrix unit $v$, let $\alpha_{v}: \widehat{r(v)} \rightarrow \mathbb{C}, \alpha_{v}(x)=\alpha\left(x, \sigma_{v}(x)\right)$. We say $\alpha$ is measurable (with respect to a measure $\mu$ ) if the functions $\alpha_{v}$ are measurable for all matrix units $v$.

If $\mu$ is a $\mathcal{G}$-quasi-invariant measure on $X$ and $\alpha$ a $\mu$-measurable 1-cocycle, there is a representation $\rho=\rho_{\mu, \alpha}$ of $\mathfrak{A}$ on $L^{2}(X, \mu)$ such that the double commutant $\rho(\mathcal{D})^{c c}$ is a masa in $\mathcal{B}\left(L^{2}(X, \mu)\right)$. Define

$$
\rho(f) \xi=f \xi, \text { and } \rho(v) \xi=\alpha_{v}\left[\frac{d \mu \circ \sigma_{v}}{d \mu}\right]^{1 / 2}\left(\xi \circ \sigma_{v}\right)
$$

$f \in \mathcal{D}(\mathcal{D}$ identified with $C(X)), \xi \in L^{2}(X, \mu)$, and $v$ a matrix unit. One notes that $\rho(v)$ is a partial isometry with initial space $d(v)=v^{*} v$ and range space $r(v)=v v^{*}$. Also, the cocycle condition implies that for matrix units $v_{1}, v_{2}$ in $\mathfrak{A}_{n}, \alpha_{v_{1} v_{2}}=\alpha_{v_{1}} \cdot \alpha_{v_{2}} \circ \sigma_{v_{1}}$, so $\rho\left(v_{1} v_{2}\right)=\rho\left(v_{1}\right) \rho\left(v_{2}\right) . \rho$ extends by linearity to a representation of $C^{*}\left(\mathfrak{A}_{n}, \mathcal{D}\right)$, and hence to $\mathfrak{A}$.

TheOREM II.1.1. Let $\mathcal{D}$ be an $S V$ masa in a unital AF algebra $\mathfrak{A}$. Then every representation $\rho$ of $\mathfrak{A}$ such that $\rho(\mathcal{D})^{c c}$ is a masa in $\mathcal{B}\left(\mathcal{H}_{\rho}\right)$ is unitarily equivalent to a representation $\rho_{\mu, \alpha}$. Two representations $\rho_{\mu, \alpha}, \rho_{\mu^{\prime}, \alpha^{\prime}}$ are unitarily equivalent if and only if the measures $\mu, \mu^{\prime}$ belong to the same equivalence class, and the 1-cocycles $\alpha, \alpha^{\prime}$ differ by a coboundary. $\rho_{\mu, \alpha}$ is irreducible if and only if $\mu$ is ergodic.

Proof. From the construction it is clear that if $\rho=\rho_{\mu, \alpha}, \rho(\mathcal{D})^{c c}$ is a masa in $\mathcal{B}\left(L^{2}(X, \mu)\right)$. Suppose $\mu, \mu^{\prime}$ are $\mathcal{G}$-quasi-invariant probability measures on $X$ and $\alpha, \alpha^{\prime}$ are 1-cocycles, and the representations $\rho=\rho_{\mu, \alpha}, \rho^{\prime}=\rho_{\mu^{\prime}, \alpha^{\prime}}$ are unitarily equivalent. Let $U: L^{2}(X, \mu) \rightarrow$ $L^{2}\left(X, \mu^{\prime}\right)$ be a unitary implementing the equivalence. Thus for each $a$ in $\mathfrak{A}, U \rho(a)=\rho^{\prime}(a) U$. For $f \in C(X) \subset L^{2}(X, \mu)$.

$$
U f=U \rho(f) 1=\rho^{\prime}(f) U 1 .=(U 1) f .
$$

Since $C(X)$ is dense in $L^{2}(X, \mu)$,

$$
U \xi=(U 1) \xi, \quad \xi \in L^{2}(X, \mu) .
$$


Since $\xi \mapsto U \xi$ is an isometry, $|U 1|=\left[\frac{d \mu}{d \mu^{\prime}}\right]^{1 / 2}$ (and so $\mu, \mu^{\prime}$ are equivalent measures); thus we may write $U 1=\gamma\left[\frac{d \mu}{d \mu^{\prime}}\right]^{1 / 2}$ where $\gamma$ is a measurable function of modulus 1 . Let $v$ be a matrix unit in $\mathfrak{A}$, $\xi \in L^{2}(X, \mu)$. Then

$$
\begin{gathered}
U \rho(v) \xi=U\left(\alpha_{v}\left[\frac{d \mu \circ \sigma_{v}}{d \mu}\right]^{1 / 2} \xi \circ \sigma_{v}\right) \\
=\gamma \alpha_{v}\left[\frac{d \mu}{d \mu^{\prime}}\right]^{1 / 2}\left[\frac{d \mu \circ \sigma_{v}}{d \mu}\right]^{1 / 2} \xi \circ \sigma_{v} \\
=\gamma \alpha_{v}\left[\frac{d \mu \circ \sigma_{v}}{d \mu^{\prime}}\right]^{1 / 2} \xi \circ \sigma_{v} \\
\rho^{\prime}(v) U \xi=\rho^{\prime}(v)\left(\gamma\left[\frac{d \mu}{d \mu^{\prime}}\right]^{1 / 2} \xi\right) \\
=\alpha_{v}^{\prime} \gamma \circ \sigma_{v}\left(\left[\frac{d \mu}{d \mu^{\prime}}\right]^{\circ} \sigma_{v}\right)^{1 / 2}\left[\frac{d \mu^{\prime} \circ \sigma_{v}}{d \mu^{\prime}}\right]^{12} \xi \circ \sigma_{v} \\
=\alpha_{v}^{\prime} \gamma \circ \sigma_{v}\left[\frac{d \mu \circ \sigma_{v}}{d \mu^{\prime} \circ \sigma_{v}}\right]^{1 / 2}\left[\frac{d \mu^{\prime} \circ \sigma_{v}}{d \mu^{\prime}}\right]^{1 / 2} \xi \circ \sigma_{v} \\
=\alpha_{v}^{\prime} \gamma \circ \sigma_{v}\left[\frac{d \mu \circ \sigma_{v}}{d \mu^{\prime}}\right]^{1 / 2} \xi \circ \sigma_{v} .
\end{gathered}
$$

Thus, $\gamma \alpha_{v}=\gamma \circ \sigma_{v} \alpha_{v}^{\prime}$; that is,

$$
\alpha_{v}^{\prime}=\gamma\left(\gamma \circ \sigma_{v}\right)^{-1} \alpha_{v} .
$$

Since the 1-cocycle $\omega(x, y)=\gamma(x) \gamma(y)^{-1}$ is a coboundary, we have shown that $\alpha, \alpha^{\prime}$ differ by a coboundary. Conversely, if $\mu, \mu^{\prime}$ are equivalent measures, and the 1-cocycles $\alpha, \alpha^{\prime}$ differ by a coboundary, the same calculation shows that $\rho_{\mu, \alpha}, \rho_{\mu^{\prime}, \alpha^{\prime}}$ are unitarily equivalent.

Suppose now that $\rho$ is a representation $\mathfrak{A}$ such that $\rho(\mathcal{D})^{c c}$ is a masa in $\mathcal{B}\left(\mathcal{H}_{\rho}\right)$. Since $\rho(\mathcal{D})$ is a direct sum of cyclic subalgebras, let $\mathcal{H}_{0} \subseteq \mathcal{H}_{\rho}$ be a closed subspace such that $\left.\rho(\mathcal{D})\right|_{\mathcal{H}_{0}}$ is cyclic. Let 
$E$ be the projection onto $\mathcal{H}_{0}$. Then $E \in \rho(\mathcal{D})^{c}$, and the reduction $\rho(\mathcal{D})^{c c} \rightarrow \rho(\mathcal{D})^{c c} E$ is an isomorphism. But $E \in \rho(\mathcal{D})^{c c}=\rho(\mathcal{D})^{c}$, which implies $E=1$. This shows $\rho(\mathcal{D})$ is cyclic.

As every cyclic representation of $\mathcal{D}$ is unitarily equivalent to multiplication on $L^{2}(X, \mu)$ for some probability measure $\mu$ on $X$ $\left(\left[7\right.\right.$, p. 49]), we may assume $\mathcal{H}_{\rho}=L^{2}(X, \mu)$ for some $\mu$, and $\rho(f) \xi=$ $f \xi, f \in \mathcal{D}$ (identified with $C(X)), \xi \in L^{2}(X, \mu)$. Using the fact that for any projection $e \in C(X)$ and matrix unit $v$, ve $v^{*}=e \circ \sigma_{v}$, we obtain that $v f v^{*}=f \circ \sigma_{v}$ for any $f \in C(X)$, as $f$ is a norm limit of linear combinations of projections. Viewing $C(X) \subset L^{2}(X, \mu)$, we have

$$
\begin{aligned}
\rho(v) f & =\rho(v) \rho(f) 1 \\
& =\rho(v) \rho\left(v^{*} v\right) \rho(f) 1 \\
& =\rho(v) \rho(f) \rho\left(v^{*} v\right) 1 \\
& =\rho\left(v f v^{*}\right) \rho(v) 1 \\
& =\rho\left(f \circ \sigma_{v}\right) \rho(v) 1 .
\end{aligned}
$$

Set $\lambda_{v}=\rho(v) 1$. Then

$$
\rho(v) f=\lambda_{v} f \circ \sigma_{v} .
$$

As $C(X)$ is dense in $L^{2}(X, \mu)$,

$$
\rho(v) \xi=\lambda_{v} \xi \circ \sigma_{v}, \quad \xi \in L^{2}(X, \mu) .
$$

Now $\rho(v)$ is a partial isometry from $d(v) L^{2}(X, \mu)$ onto $r(v) L^{2}(X, \mu)$. If $\xi$ is any vector in $d(v) L^{2}(X, \mu)$ then $\|\rho(v) \xi\|=\|\xi\|$. Thus

$$
\begin{aligned}
\|\rho(v) \xi\|^{2} & =\int\left|\lambda_{v}\right|^{2}\left|\xi \circ \sigma_{v}\right|^{2} d \mu \\
& =\int\left|\lambda_{v} \circ \sigma_{v}^{-1}\right|^{2}|\xi|^{2} d \mu \circ \sigma_{v}^{-1} \\
& =\int|\xi|^{2} d \mu \\
& =\|\xi\|^{2} .
\end{aligned}
$$

It follows

$$
\left|\lambda_{v} \circ \sigma_{v}^{-1}\right|=\left[\frac{d \mu \circ \sigma_{v}^{-1}}{d \mu}\right]^{-1 / 2}
$$


and in particular that $\mu \circ \sigma_{v}^{-1}, \mu$ are equivalent measures (as measures on $\widehat{d(v)})$. Now $\sigma_{v}^{-1}=\sigma_{v^{*}}$ and as $v$ was an arbitrary matrix unit, we have that $\mu$ is $\mathcal{G}$ - quasi-invariant. The fact that $\rho\left(v_{1} v_{2}\right)=$ $\rho\left(v_{1}\right) \rho\left(v_{2}\right)$ from matrix units $v_{1}, v_{2}$ implies $\lambda_{v_{1} v_{2}}=\lambda_{v_{1}} \lambda_{v_{2}} \circ \sigma_{v_{1}}$. If $e$ is a projection in $\mathcal{D}, \lambda_{e}=\rho(e)$. Taking $v_{1}=v^{*}, v_{2}=v$, get

$$
\rho\left(v^{*} v\right)=\lambda_{v^{*}} \lambda_{v} \circ \sigma_{v^{*}}
$$

Since the functions on the right are supported on $\widehat{d(v)}$, we have $\lambda_{v^{*}}=\left(\lambda_{v} \circ \sigma_{v^{*}}\right)^{-1}$ on $\widehat{d(v)}$, or

$$
\lambda_{v^{*}}=\left(\lambda_{v} \circ \sigma_{v}^{-1}\right)^{-1} .
$$

From the above we obtain

$$
\left|\lambda_{v^{*}}\right|=\left[\frac{d \mu \circ \sigma_{v}^{-1}}{d \mu}\right]^{1 / 2}=\left[\frac{d \mu \circ \sigma_{v^{*}}}{d \mu}\right]^{1 / 2} .
$$

Replacing $v^{*}$ by $v$,

$$
\left|\lambda_{v}\right|=\left[\frac{d \mu \circ \sigma_{v}}{d \mu}\right]^{1 / 2}
$$

Express $\lambda_{v}=\alpha_{v}\left[\frac{d \mu \circ \sigma_{v}}{d \mu}\right]^{1 / 2}$; then $\alpha_{v}$ is a $\mu$-measurable function of modulus one. Furthermore, the equation $\lambda_{v_{1} v_{2}}=\lambda_{v_{1}} \lambda_{v_{2}} \circ \sigma_{v_{1}}$ implies $\alpha_{v_{1} v_{2}}=\alpha_{v_{1}} \alpha_{v_{2}} \circ \sigma_{v_{1}}$. Thus the functions $\left\{\alpha_{v}\right\}, v$ a matrix unit, define a 1-cocycle $\alpha$, and we have shown that $\rho$ is unitarily equivalent to $\rho_{\mu, \alpha}$.

Suppose $\rho=\rho_{\mu, \alpha}$ with $\mu$ ergodic, and let $T=T^{*} \in \rho(\mathfrak{A})^{c}$. As $\rho(\mathfrak{A})^{c}$ is generated by its self-adjoint elements, it is enough to show $T$ is a scalar. Writing $T=\int_{-\|T\|}^{\|T\|} t d E_{t}$ the spectral decomposition, the spectral projections $E_{t}$ commute with all operators commuting with $T$, so $E_{t} \in \rho(\mathfrak{A})^{c}$. As $E_{t} \in \rho(\mathcal{D})^{c}=\rho(\mathcal{D})^{c c}$, for any matrix unit $v$ we have

$$
\rho\left(v v^{*}\right) E_{t}=\rho(v) E_{t} \rho\left(v^{*}\right)=E_{t} \circ \sigma_{v} .
$$

It follows that the support $\widehat{E}_{t}$ is $\mathcal{G}$-invariant, so that $\mu\left(\widehat{E}_{t}\right)=0$ or 1 . Thus $E_{t}=0$ or 1 for all $t$, so $T$ is a scalar. Conversely, if $\mu$ is not ergodic and $\widehat{E}$ is a $\mathcal{G}$-invariant subset of $X$ with $0<\mu(\widehat{E})<1$, then the corresponding projection $E$ is reducing. 
REMarK II.1.2. Stratila and Voiculescu studied the representations $\rho_{\mu}=\rho_{\mu, 1}$ (in our notation) for which the cocycle is the constant $1\left[14\right.$, p. 52]. Henceforth we will also write $\rho_{\mu}$ in place of $\rho_{\mu, 1}$.

Definition II.1.3. Let $\mathfrak{A}$ be an $\mathrm{AF}$ algebra and $\mathcal{D} \subset \mathfrak{A}$ a distinguished masa. A representation $\rho$ of $\mathfrak{A}$ in $\mathcal{B}\left(\mathcal{H}_{\rho}\right)$ is called $\mathcal{D}$ preserving or masa-preserving if the weak closure of $\rho(\mathcal{D})$ is a masa in $\mathcal{B}\left(\mathcal{H}_{\rho}\right)$. If $\mathcal{T} \subset \mathfrak{A}$ is a TAF algebra, then $\rho$ is masa-preserving if it is $\mathcal{D}$-preserving for the diagonal masa $\mathcal{D}=\mathcal{T} \cap \mathcal{T}^{*}$.

Note that if $\rho$ is $\mathcal{D}$-preserving, and $\mathcal{E} \subset \mathfrak{A}$ is another masa, $\rho$ may not be $\mathcal{E}$-preserving.

II.2. Properties of masa-preserving representations. In what follows, it will be convenient to work with invariant measures when possible. Thus, quasi- invariant probability measures are in some cases replaced by equivalent, $\sigma$-finite, invariant measures.

If $\mathcal{T} \subset \mathfrak{A}$ is a TAF subalgebra with $\mathcal{T} \cap \mathcal{T}^{*}=\mathcal{D}$, say a subset $Y \subset X$ is decreasing if, whenever $y \in Y$ and $x \prec y$, then $x \in Y$. Let $\tilde{\mathcal{T}}$ be the weak closure of $\rho(\mathcal{T})$ in $\mathcal{B}(\mathcal{H})$. Finally, let $P_{E}$ be the projection $\xi \mapsto \chi_{E} \xi$ for $E \subset X$ measurable.

Proposition II.2.1. Let $\mathcal{T}$ be a TAF subalgebra of the AF algebra $\mathfrak{A}$ with diagonal $\mathcal{D}, \rho: \mathfrak{A} \rightarrow \mathcal{B}(\mathcal{H})$ a masa-preserving representation. Letting $\tilde{\mathcal{T}}=\rho \overline{(\mathcal{T})}^{\mathrm{wk}}$, and $\mathcal{L}=\operatorname{Lat}(\tilde{\mathcal{T}})$ we have

(i) $\mathcal{L}=\left\{P_{D}: D \subset X, D\right.$ measurable, decreasing $\}$. In other words $\mathcal{L}$ is a CSL with Arveson representation given by the triple $(X, \prec, \mu)$, where $\mu$ is the measure associated with $\rho$.

(ii) If $\mathcal{L}$ is a nest, then $\tilde{\mathcal{T}}=\operatorname{Alg}(\mathcal{L})$.

Proof. (i) We may suppose by Theorem 1 that $\rho=\rho_{\mu, \alpha}$. Since $\tilde{\mathcal{T}} \supset \widetilde{\mathcal{D}}=L^{\infty}(X, \mu)$, any subspace invariant under $\widetilde{\mathcal{T}}$ is of the form $P_{E} \cdot L^{2}(X, \mu)$ for some measurable $E \subset X$. If $\xi \in L^{2}(X, \mu)$ is supported on $E, v$ a matrix unit in $\mathcal{T}$, then $\rho(v) \xi$ is supported on $\sigma_{v}^{-1}(E)=\sigma_{v}^{-1}\left(E \cap \widehat{v^{*} v}\right)$. Thus, $\sigma_{v}^{-1}(E) \subset E$, for each matrix unit $v$ in $\mathcal{T}$. In other words, $E$ is decreasing.

(ii) Follows immediately from [3, Cor. 15.12].

Note that in the above proposition, $\mathcal{T}$ is not assumed to be strongly maximal. 
If $\rho: \mathfrak{A} \rightarrow \mathcal{B}(\mathcal{H})$ is a representation, and $\mathcal{T} \subset \mathfrak{A}$ is TAF, is it true that $\tilde{\mathcal{T}}=\overline{\rho(\mathcal{T})}^{\text {wk }}$ is a CSL algebra? In other words, when is $\tilde{\mathcal{T}}$ synthetic in the sense of Arveson's work [1, Definition 2.2.1]?

In light of Examples I.1, 2 and 4, D. Larson raised the question of whether every strongly maximal triangular subalgebra of a UHF algebra can be represented as a weakly dense subalgebra of a nest algebra in which the nest is maximal. The next proposition answers this question in the affermative; in fact, the result is true for a much broader class of AF algebras, namely the primitive AF algebras. Recall that a $C^{*}$-algebra is primitive if it has a faithful irreducible representation; in particular, if $\mathfrak{A}$ is simple then $\mathfrak{A}$ is primitive. From $[7,3.13 .10 ; 4.3 .6]$ and

$[12$, II.4.6] $\mathfrak{A}$ is primitive if $X$ has a dense orbit. This fact is used below.

Proposition II.2.2. Let $\mathfrak{A}$ be a primitive $A F$ algebra, $\mathcal{T} \subset \mathfrak{A}$ a strongly maximal TAF-subalgebra. Then there is a faithful, irreducible masa-preserving representation $\rho$ of $\mathfrak{A}$ such that $\tilde{\mathcal{T}}=\rho \overline{(\mathcal{T})}$ wk is a triangular nest algebra in $\mathcal{B}\left(\mathcal{H}_{\rho}\right)$.

Proof. Let $\mathcal{D}=\mathcal{T} \cap \mathcal{T}^{*}$ and $X$ the Gelfand spectrum of $\mathcal{D}$. By the above remarks, $X$ has a dense orbit, $\left[x_{0}\right]$. Since $\mathcal{T}$ is strongly maximal, the orbit $\left[x_{0}\right]$ is totally ordered. Let $\mu$ be counting measure on $\left[x_{0}\right]$ : thus if $E \subset X, \mu(E)=\operatorname{card}\left(E \cap\left[x_{0}\right]\right)$. Note that $\mu$ is a $\sigma$-finite Borel measure, which is invariant and ergodic. Thus the representation $\rho_{\mu}: \mathfrak{A} \rightarrow \mathcal{B}\left(\mathcal{H}_{\rho}\right)$ is irreducible. As the orbit $\left[x_{0}\right]$ is totally ordered, the decreasing subsets of $\left[x_{0}\right]$ are totally ordered. Every projection $P$ in $L^{\infty}(X, \mu)$ is of the form $P=P_{E}$, with $E \subset$ $\left[x_{0}\right]$, and it follows from Proposition 2.1 that Lat $\tilde{\mathcal{T}}$ is a nest, $\mathcal{N}$. Also, from (ii), $\tilde{\mathcal{T}}=\operatorname{Alg}(\mathcal{N})$.

It will follow that $\tilde{\mathcal{T}}$ is triangular if $\mathcal{N}$ is maximal; to see this is true, let $\left[x_{0}\right]=\left\{x_{i}\right\}_{i \in I}$, where the index set $I$ is ordered so that $x_{i} \prec x_{j}$ iff $i \leq j$. Let $P_{i}$ be the one-dimensional projection onto the span of $\xi_{i} \in L^{2}(X, \mu)=\mathcal{H}_{\rho}$, where

$$
\xi_{i}\left(x_{j}\right)= \begin{cases}1, & i=j \\ 0, & \text { otherwise }\end{cases}
$$


It is enough to show each $P_{i} \in \mathcal{N}^{c c}$ for then $N^{c c}=L^{\infty}(X, \mu)$. Let

$$
D_{i}=\left\{x_{j}: j \leq i, j \neq i\right\} \quad \text { and } \quad D_{i}^{+}=\left\{x_{j}: j \leq i\right\}=D_{i} \cup\left\{x_{i}\right\}
$$

Then $D_{i}, D_{i}^{+}$are both decreasing, so

$$
P_{D_{i}}, P_{D_{i}^{+}} \in \mathcal{N}, \quad \text { and } \quad P_{i}=P_{D_{i}^{+}}-P_{D_{i}}
$$

To show $\rho$ is faithful, note that $\operatorname{ker} \rho$ is an ideal in $\mathcal{A}$, and hence ker $\rho$ is the norm-closed $\mathcal{D}$-module generated by its matrix units. If $v$ is a matrix unit with $\rho(v)=0$, then $\rho\left(v^{*} v\right)=0$. Thus it suffices to show $\rho(e) \neq 0$ for $e$ a projection in $\mathcal{D}$. But if $e$ is a nonzero projection in $\mathcal{D}$, supp $e=\hat{e}$ is a nonempty open set in $X$, and since $\left\{x_{i}\right\}_{i \in I}$ is dense, there is some $x_{j} \in \hat{e}$. Then $\rho(e) \xi_{i} \neq 0$.

II.3. Examples of measure-cocycle constructions. Now we return to the standard embedding algebra $\mathcal{T}=\underline{\lim _{\rightarrow}}\left(\mathcal{T}_{n}, \sigma_{n}\right)$ of Example I. 2 to see that the representation described there is of the type $\rho_{\mu}$. If the standard embedding is is given in binary notation i.e.,

$$
\begin{aligned}
\sigma_{n}\left(e_{\left(i_{0}, \ldots, i_{n-1}\right),\left(j_{0}, \ldots, j_{n-1}\right)}^{(n)}\right)= & e_{\left(i_{0}, \ldots, i_{n-1}, 0\right),\left(j_{0}, \ldots, j_{n-1}, 0\right)}^{(n+1)} \\
& +e_{\left(i_{0}, \ldots, i_{n-1}, 1\right),\left(j_{0}, \ldots, j_{n-1}, 1\right)}^{(n+1)}
\end{aligned}
$$

then the sequences $\left(i_{0}, \ldots, i_{n-1}\right),\left(j_{0}, \ldots, j_{n-1}\right)$ are identified with the integers $1+\sum_{i=0}^{n-1} i_{\ell} 2^{\ell}, 1+\sum_{\ell=0}^{n-1} j_{\ell} 2^{\ell}$ respectively. From this it is easy to see that two points $x, y \in X=\prod_{n=0}^{\infty}\{0,1\}_{n}$ are in the same orbit iff they have the same tails, and in that case the ordering is given by $x \prec y$ if either $x=y$ or else $\exists N \in \mathbb{N}$ with $x_{N}<y_{N}$ and $x_{n}=y_{n}, \quad n>N$. Thus the ordering on each orbit ("reverse lexicographic order") has the property that each element except for $\overline{1}=(1,1,1, \ldots)$ has an immediate successor, and each element except for $\overline{0}=(0,0,0, \ldots)$ has an immediate predecessor. (See $[\mathbf{1 6}]$, or [4] for a fuller discussion.) In particular the orbit [0] is orderisomorphic to the natural numbers.

Thus the Smith representation of $\mathcal{T}$ on $\ell^{2}(\mathbb{N})$ is obtained by choosing $\mu$ to be counting measure on the orbit $[\overline{0}]$; note that the formula 
for $\rho\left(e_{i j}^{(n)}\right) \xi_{\ell}$ can be expressed in binary form as

$$
\begin{aligned}
& \rho\left(e_{\left(i_{0}, \ldots, i_{n-1}\right),\left(j_{0}, \ldots, j_{n-1}\right)}^{(n)}\right) \xi_{\ell_{0}, \ldots, \ell_{n-1}, \ell_{n}, \ell_{n+1}, \ldots} \\
& \quad= \begin{cases}\xi_{\left(i_{0}, \ldots, i_{n-1}, \ell_{n}, \ell_{n+1}, \ldots\right)} & \text { if }\left(\ell_{0}, \ldots, \ell_{n-1}\right)=\left(j_{0}, \ldots, j_{n-1}\right) \\
0, & \text { otherwise. }\end{cases}
\end{aligned}
$$

But this is precisely $\rho_{\mu}$. The projections $P_{\langle 1, \ldots, n\rangle}$ of I.2 correspond to projections onto descreasing sets, so the assertions of I.2 follow from Proposition II.2.

The representations in Examples I.1, I.3 and I.4 are also of the form $\rho_{\mu}$, but for $\mu$ non-discrete. For the canonical nest algebra $\left.\tilde{\mathcal{T}}=\varliminf_{\left(\mathcal{T}_{n}\right.}, \nu_{n}\right)$ in I.1, the Gelfand spectrum $X$ of the diagonal can be identified with $\prod_{n=1}^{\infty}\{0,1\}_{n}$. Two points $x, y \in X$ belong to the same orbit if they have the same tails, and the ordering on each orbit is lexicographic: $\left(x_{n}\right) \prec\left(y_{n}\right)$ if either $x=y$ or for some $N, x_{N}<y_{N}$, and $x_{n}=y_{n}$ for $1 \leq n<N$. Let $\mu_{n}$ be the measure on $\{0,1\}$, $\mu_{n}(0)=\mu_{n}(1)=1 / 2$, and $\mu=\prod_{n=1}^{\infty} \mu_{n}$, the product measure. Also, note that the map $X \rightarrow[0,1],\left(x_{n}\right) \rightarrow \sum_{n=1}^{\infty} x_{n} 2^{-n}$, gives a measurespace isomorphism of $(X, \mu)$ with $([0,1], m)$. Let $\left\{e_{\left(i_{1}, \ldots, i_{n}\right),\left(j_{1}, \ldots, j_{n}\right)}^{(n)}\right\}$, $i_{\ell}, j_{\ell} \in\{0,1\}, 1 \leq \ell \leq n$, be a system of matrix units for $\mathfrak{A}_{n}$, indexed binarily, and satisfying

$$
\begin{aligned}
\nu_{n}\left(e_{\left(i_{1}, \ldots, i_{n}\right),\left(j_{1}, \ldots, j_{n}\right)}^{(n)}\right) & =e_{\left(i_{1}, \ldots, i_{0}, 0\right),\left(j_{1}, \ldots, j_{n}, 0\right)}^{(n+1)}+e_{\left(i_{1}, \ldots, i_{n}, 1\right),\left(j_{i}, \ldots, j_{n}, 1\right)}^{(n+1)} .
\end{aligned}
$$

The relation between binary and integer indexing is $\left(i_{1}, \ldots, i_{n}\right) \rightarrow$ $\sum_{\ell=1}^{n} i_{\ell} 2^{n-\ell}$. The map $x=\left(x_{n}\right)_{n=1}^{\infty} \rightarrow \sum_{n=1}^{\infty} x_{n} 2^{-n}$ implements an isomorphism of $L^{2}(X, \mu)$ and $L^{2}([0,1], m)$, under which $\rho_{\mu}\left(e_{\left.i_{1}, \ldots, i_{n}\right),\left(j_{1}, \ldots, j_{n}\right)}^{(n)}\right)$ is transformed into the operator $\rho\left(e_{i j}^{(n)}\right)$ of I.1, where

$$
\left(i_{1}, \ldots, i_{n}\right) \rightarrow i,\left(j_{1}, \ldots, j_{n}\right) \rightarrow j .
$$

The fact $\mu$ is invariant is equivalent to the invariance of Lebesgue measure under dyadic translations. The ergodicity of $\mu$ can be obtained from the fact that $m$ is the unique translation-invariant measure on $[0,1]$, or directly as $\mu$ is the measure on $X$ associated with the unique normalized trace on the diagonal. 
The Hilbert space isomorphism $L^{2}(X, \mu) \rightarrow L^{2}([0,1], m)$ of the previous paragraph also yields an equivalence of the representation $\rho$ of Example I.3 with a masa-preserving representation $\rho_{\mu}$ of the standard embedding algebra. This $\rho_{\mu}$ is a particular instance of Proposition III.2.1. ([16] contains a useful discussion of the standard embedding TAF algebra.)

For Example I.4, view $X$ as described in [4]: $X=\prod_{n=-\infty}^{\infty}\{0,1\}_{n}$, where $x, y \in X$ belong to the same orbit if they have the same tails (both to the left and to the right), and $x \prec y$ if for some $N$, $x_{N} \leq y_{N}$, and $x_{n}=y_{n}$ for $n<N$. Let $X_{-n} \subset X$ be the set of points $x=\left(x_{k}\right)_{k=-\infty}^{\infty}$ with $x_{k}=0, k<-n$. Define a measure $\mu$ on $\bigcup_{n=0}^{\infty} X_{-n}$ by

$$
\mu\left\{x: x \in X_{-n}, x_{-n}=\ell_{-n}, \ldots, x_{m}=\ell_{m}\right\}=2^{-m}
$$

for each integer $m, m \geq-n$, and each choice of $\ell_{-n}, \ldots, \ell_{m}$. Set $\mu\left(X \backslash \bigcup_{n=0}^{\infty} X_{-n}\right)=0 . \quad$ Let $\Lambda: \bigcup_{n=0}^{\infty} X_{-n} \rightarrow[0, \infty)$, by $\Lambda\left(x_{0}\right)=\sum_{\ell=-\infty}^{\infty} x_{\ell} 2^{-\ell}$. This is well defined as $x_{\ell}=0$ for $-\ell$ sufficiently large. $\Lambda$ gives an isomorphism of the measure spaces $(X, \mu)$ and $([0, \infty), m)$. We leave it to the reader to verify that $\mu$ is an invariant ergodic $\sigma$-finite measure on $X$, and that $\Lambda$ implements a unitary equivalence of the representation $\rho$ of Example I.4 and the representation $\rho_{\mu}$ on $L^{2}(X, \mu)$. Since $\rho_{\mu}$ is masa-preserving, it follows from Theorem II.1.1 that $\rho_{\mu}$ is irreducible, and from Proposition 0.1 that $\rho_{\mu}(\mathcal{T})$ is weakly dense in a nest algebras.

\section{Attainable order types.}

\section{III.1. Algebras in which Lat $\mathcal{T}$ generates the diagonal.}

Proposition III.1.1. Suppose $\mathcal{T}$ is a strongly maximal TAF subalgebra of $\mathfrak{A}$ and $\rho$ is a weakly dense representation of $\mathfrak{A}$ in $\mathcal{B}\left(\mathcal{H}_{\rho}\right)$ such that the weak closure of $\rho(\mathcal{T})$ contains a masa. If the invariant projections of $\mathcal{T}$ in $\mathfrak{A}$ generate the diagonal of $\mathcal{T}$ as a $C^{*}$-algebra, then Lat $\rho(\mathcal{T})$ is either a continuous nest or else it is a totally 
atomic nest whose atoms are ordered as one of the orbits in the fundamental relation.

Proof. If Lat $\rho(\mathcal{T})$ has no atoms then it is continuous, so suppose it has an atom $E$. Then $E \rho(t) E$ is a homomorphism on $\mathcal{D}$ which maps every projection in $\mathcal{T}$ to either $E$ or 0 . Since the invariant projections of $\mathcal{T}$ generate all the projections of $\mathcal{D}$ this means every projection in $\mathcal{D}$ is mapped to 0 or $E$. $\mathcal{D}$ is the closed linear span of its projections, and so $E \rho E$ is an element of $\widehat{\mathcal{D}}$. (Also, the irreducibility implies that $E$ is rank-one.)

Let

$$
\begin{array}{r}
F=\vee\left\{\rho\left(e_{i, j}^{(n)}\right) E \rho\left(e_{j, i}^{(n)}\right):(i, j)\right. \text { for which } \\
\left.e_{i, j}^{(n)} \text { is defined, } n=1,2, \ldots\right\} .
\end{array}
$$

Then $F$ is an invariant projection for all $\rho\left(e_{i, j}^{(n)}\right)$ and hence for $\mathfrak{A}$. Since $\rho$ is an irreducible representation of $\mathfrak{A}, F$ is the identity. Thus Lat $\rho(\mathcal{T})$ is totally atomic.

The ordering of the atoms of Lat $\rho(\mathcal{T})$ is induced by the same conjugations that induce the ordering of the equivalence class of $E$, viewed as an element of $\widehat{\mathcal{D}}$.

It also follows from the proof that if the nest is not continuous, the von Neumann algebra generated by the atams, which are rankone, is a masa, and hence the image of the diagonal is weakly dense in a masa. We have proved

Corollary III.1.2. If $\mathcal{T}, \rho$ are as above, then either Lat $\rho(\mathcal{T})$ is a continuous nest, or else $\rho$ is masa-preserving.

If $\mathcal{T}$ is the refinement algebra, then between any two points on an orbit in the Gelfand space of the diagonal lies a third point. Thus

CoROllary III.1.3. If $\rho$ is an irreducible representation of the $U H F$ algebra $\mathfrak{A}$ and $\mathcal{T} \subset \mathfrak{A}$ is the refinement algebra, then Lat $\rho(\mathcal{T})$ is either a continuous nest, or else it is a Cantor nest of multiplicity one.

If Lat $\rho(\mathcal{T})$ is a continuous nest, then $\rho$ need not be masapreserving. In Section IV.3 we will present an irreducible representation $\rho$ of the ambient UHF algebra of the refinement algebra 
$\mathcal{T}$ such that Lat $\rho(\mathcal{T})$ is a continuous nest of uniform multiplicity two. On the other hand, if $\rho$ is masa-preserving, we obtain

Corollary III.1.4. Let $\mathfrak{A}, \mathcal{T}, \rho$ be as in Proposition 1.1. Suppose in addition that $\rho$ is masa-preserving. Then $\tilde{\mathcal{T}}=\rho \overline{(\mathcal{T})}^{\text {wk }}$ is a triangular subalgebra of $\mathcal{B}\left(\mathcal{H}_{\rho}\right)$.

Proof. If Lat $\tilde{\mathcal{T}}$ is totally atomic with rank-one atoms, then $\tilde{\mathcal{T}}=$ $\operatorname{Alg}($ Lat $\tilde{\mathcal{T}})$ is triangular. Otherwise, Lat $\tilde{\mathcal{T}}$ is a continuous nest of multiplicity one since $\rho$ is masa-preserving, and in this case $\tilde{\mathcal{T}}$ is also triangular.

III.2. Representations of the standard algebra. In this section $\mathcal{T}$ will denote the standard embedding algebra in the UHF $\left(2^{\infty}\right)$ algebra $\mathfrak{A}$, and we will completely describe the order types of the nests in which $\mathcal{T}$ can be densely represented. Here we drop the condition that the representation $\pi$ satisfy $\pi{\overline{\left(\mathcal{T} \cap \mathcal{T}^{*}\right)}}^{\text {wk }}$ be a masa in $\mathcal{B}\left(\mathcal{H}_{\pi}\right)$, and only assume $\pi$ be $*$-extendible.

THEOREM III.2.1. Let $\pi$ be an irreducible representation of $\mathfrak{A}$ such that $\pi \overline{(\mathcal{T})}^{\text {wk }}$ contains a masa. Then $\mathcal{N}=$ Lat $\pi(\mathcal{T})$ is either a two point lattice with a single infinite rank atom, or else is a multiplicity-free nest ordered as a subset of the integers.

Examples I.3 and I.2, together with obvious modifications, show that all of these lattices are attained by representations.

We need first a couple of lemmas.

Lemma III.2.2. Let $\pi, \mathcal{T}$ be as above. Then for each $n \in \mathcal{N}$ other than $0, I$ there is a $k$ and $1 \leq i<j \leq 2^{k}$ such that

$$
\pi\left(e_{i, j}^{(k)}\right) n \pi\left(e_{i, j}^{(k)}\right)^{*}<\pi\left(e_{i i}^{(k)}\right) n
$$

Proof. Note that since $\pi\left(e_{i, j}^{(k)}\right) \pi\left(e_{i, j}^{(k)}\right)^{*}=\pi\left(e_{i, i}^{(k)}\right)$ belongs to the commutant of $\mathcal{N}, \pi\left(e_{i, j}^{(k)}\right) n \pi\left(e_{i, j}^{(k)}\right)^{*}$ is a projection and since 
$\pi\left(e_{i, j}^{(k)}\right)$ is in $\operatorname{Alg} \mathcal{N}$ it is dominated by both $n$ and $\pi\left(e_{i, i}^{(k)}\right)$. Thus suppose, on the contrary that for each choice of $k, i, j$,

$$
\pi\left(e_{i, j}^{(k)}\right) n \pi\left(e_{i j}^{(k)}\right)^{*}=\pi\left(e_{i, i}^{(k)}\right) n .
$$

Then since $n$ and $\pi\left(e_{i, i}^{(k)}\right)$ commute, $\pi\left(e_{i, j}^{(k)}\right)$ and $n$ would commute and $n$ would reduce $\pi(\mathcal{T})$.

LEMMA III.2.3. Let $v=\pi\left(e_{i, j}^{(k)}\right)$ for some $k$ and $1 \leq i<j \leq 2^{k}$ and let $p_{1}=v v^{*}, p_{2}=v^{*} v$. Let $n \in \mathcal{N}$. If $v n v^{*}<p_{1} n$ then there is an $n^{\prime} \in \mathcal{N}$ with $n^{\prime}<n$ and $n-n^{\prime} \perp p_{2}$.

Proof. As before, $p_{1}$ and $n$ commute and $v n v^{*}$ is a projection. Let $q_{1}=p_{1} n-v n v^{*}$ and $q_{2}=p_{2} n$. Now we claim that because $\mathcal{T}$ is a limit of standard embeddings, $e_{i, i}^{(k)} \mathcal{T} e_{j, j}^{(k)}=e_{i, j}^{(k)} \mathcal{T} e_{j, j}^{(k)}$. Observe that for any $k^{\prime}>k$,

$$
e_{i, i}^{(k)}=\sum_{r=0}^{2^{\left(k^{\prime}-k\right)}-1} e_{i+r 2^{k}, i+r 2^{k}}^{\left(k^{\prime}\right)}
$$

Thus for any matrix unit $v$ in $\mathcal{T}_{k^{\prime}}$, either $e_{i, i}^{(k)} v e_{j, j}^{(k)}$ is zero or else $v=$ $e_{i+r 2^{k}, j+s 2^{k}}^{\left(k^{\prime}\right)}$ for some $i+r 2^{k} \leq j+s 2^{k}$. Now $(s-r) 2^{k} \geq i-j>-2^{k}$ so $s \geq r$. Thus,

$$
\begin{aligned}
v & =e_{i+r 2^{k}, j+r 2^{k}}^{\left(k^{\prime}\right)} e_{j+r 2^{k}, j+s 2^{k}}^{\left(k^{\prime}\right)} \\
& =e_{i+r 2^{k}, j+r 2^{k}}^{\left(k^{\prime}\right)} e_{j+r 2^{k}, j+r 2^{k}}^{\left(k^{\prime}\right)} e_{j+r 2^{k}, j+s 2^{k}}^{\left(k^{\prime}\right)} \\
& =e_{i, j}^{(k)} e_{j+r 2^{k}, j+s 2^{k}}^{\left(k^{\prime}\right)}
\end{aligned}
$$

Thus, the claim follows. Now note that

$$
\begin{aligned}
q_{1} \pi(\mathcal{T}) q_{2} & =q_{1} \pi\left(e_{i, i}^{(k)} \mathcal{T} e_{j, j}^{(k)}\right) q_{2} \\
& =q_{1} \pi\left(e_{i, j}^{(k)} \mathcal{T} e_{j, j}^{(k)}\right) q_{2} \\
& =q_{1} v n \pi(\mathcal{T}) q_{2} \\
& =0
\end{aligned}
$$

Thus $q_{1} \operatorname{Alg} \mathcal{N} q_{2}=0$ and so we must have an $n^{\prime} \in \mathcal{N}$ such that

$$
q_{2} \leq n^{\prime} \perp q_{1}
$$


But $q_{1}$ is a non-zero projection dominated by $n$ and so $n>n^{\prime}$. On the other hand, $0 \leq p_{2}\left(n-n^{\prime}\right)=q_{2}-p_{2} n^{\prime} \leq 0$ so $p_{2} \perp(n-$ $n^{\prime}$ ).

Proof of III.1.1. By Proposition 0.1, $\pi(\mathcal{T})$ is weakly dense in $\operatorname{Alg} \mathcal{N}$, where $\mathcal{N}$ is a nest. We shall show that every $n$ other than 0 or 1 in $\mathcal{N}$ has an immediate predecessor. Since there exists a conjugate-linear automorphism of $T$ and the adjoint map maps $\operatorname{Alg} \mathcal{N}$ to $\operatorname{Alg} \mathcal{N}^{\perp}$, which is the reversed order structure, it is clear that this will also show that every element in $\mathcal{N}$ other than $I$ must have an immediate successor.

Suppose $n \in \mathcal{N} \backslash\{0,1\}$ has no immediate predecessor. Now, by Lemma $\mathrm{A}$, we can choose $k$ and $1 \leq i<j \leq 2^{k}$ such that $\pi\left(e_{i, j}^{(k)}\right) n \pi\left(e_{i, j}^{(k)}\right)^{*}<\pi\left(e_{i, i}^{(k)}\right) n$. Then by Lemma 3 , there is an $n^{\prime}<$ $n$ such that $\left(n-n^{\prime}\right) \perp \pi\left(e_{j, j}^{(k)}\right)$. But then the map $\hat{\pi}(t)=(n-$ $\left.n^{\prime}\right) \pi(t)\left(n-n^{\prime}\right)$ is a homomorphism on $\mathcal{T}$. Moreover, $\hat{\pi}\left(e_{j, j}^{(k)}\right)=0$. Thus, for any $l \geq 0$

$$
\hat{\pi}\left(e_{j+r 2^{k}, j+r 2^{k}}^{(k+l)}\right)=0
$$

for $r=0, \ldots, 2^{l}-1$.

Since, if $0 \leq r<2^{l}$ and $i^{\prime} \leq j+r 2^{k} \leq j^{\prime}$ then

$$
e_{i^{\prime}, j^{\prime}}^{(k+l)}=e_{i^{\prime}, j+r 2^{k}}^{(k+l)} e_{j+r 2^{k}, j+r 2^{k}}^{(k+l)} e_{j+r 2^{k}, j^{\prime}}^{(k+l)}
$$

it follows that $\hat{\pi}\left(e_{i^{\prime}, j^{\prime}}^{\left(k^{\prime}\right)}\right)=0$ whenever $k^{\prime}>k$ and $j^{\prime}-i^{\prime} \geq 2^{k}$. Thus, for $v \in \mathcal{T}_{k^{\prime}}$

$$
\hat{\pi}\left(\sum_{r=1}^{2^{k}} e_{r, r}^{(k)} v e_{r, r}^{(k)}\right)=\hat{\pi}\left(\sum_{r=1}^{2^{k^{\prime}}} e_{r, r}^{\left(k^{\prime}\right)} v e_{r, r}^{\left(k^{\prime}\right)}\right)
$$

which belongs to the $\pi\left(\mathcal{T} \cap \mathcal{T}^{*}\right)$. Since this holds for all $k^{\prime}>k$, it follows that, taking $p_{r}=\hat{\pi}\left(e_{r, r}^{(k)}\right)$,

$$
\sum_{r} p_{r} \pi(v) p_{r}
$$

belongs to $\mathcal{N}^{c}$ for all $v \in \mathcal{T}$. But thus, $p_{r} \pi(\mathcal{T}) p_{r} \subseteq \pi\left(\mathcal{T} \cap \mathcal{T}^{*}\right)$ for $1 \leq r \leq 2^{k}$.

Since also $p_{r} \pi\left(\mathcal{T}^{*}\right) p_{r} \subseteq \pi\left(\mathcal{T} \cap \mathcal{T}^{*}\right)$ and $\mathcal{T}$ is strongly maximal triangular, $p_{r} \pi(\mathfrak{A}) p_{r} \subseteq \pi\left(\mathcal{T} \cap \mathcal{T}^{*}\right)$. But $\pi\left(\mathcal{T} \cap \mathcal{T}^{*}\right)$ is abelian, hence 
$p_{r}$ is rank-one. Since $\sum_{r=1}^{2^{k}} p_{r}=n-n^{\prime}, n$ has an immediate predecessor. Moreover, if $n-$ is the immediate predecessor of $n$ then $(n-n-) \pi(n-n-)$ gives an irreducible, finite dimensional representation of $\mathcal{T}$, which requires that $n-n-$ be rank-one. Finally, since $\mathfrak{A}$ is simple it has no finite dimensional representations and $\mathcal{H}_{\pi}$ is infinite dimensional. Thus either $\mathcal{N}=\{0,1\}$ or else $\mathcal{N}$ is infinite (since its atoms are rank-one).

III.3. Masa-preserving representations of $\mathbb{Z}$-analytic algebras. From [14], given an AF algebra $\mathfrak{A}=\overline{\cup \mathfrak{A}_{n}}$ and masa $\mathcal{D}$ with $\mathcal{D} \cap \mathfrak{A}_{n}$ a masa in $\mathfrak{A}_{n}$, one constructs a conditional expectation $\mathcal{E}: \mathfrak{A} \rightarrow \mathcal{D}$, with the property that $\mathcal{E}\left(v a v^{*}\right)=v \mathcal{E}(a) v^{*}$ for any matrix unit $v, a \in \mathfrak{A}$. (Matrix units are chosen as described at the beginning of this section.) It follows that there is a one- to-one correspondence between tracial states on $\mathfrak{A}$ and invariant probability measures on $X=\mathcal{D}^{\wedge}$, given by

$$
\tau(a)=\int_{X} \mathcal{E}(a)(t) d \mu_{\tau}(t),
$$

where $\tau$ is a tracial state, $\mu_{\tau}$ an invariant probability measure. Indeed, given $\tau,\left.\tau\right|_{\mathcal{D}}$ is a positive, invariant norm-one linear functional, hence corresponds to an invariant probability measure. Conversely, given such a measure $\mu$, the formula defines a tracial state $[14$, p. 33]. Under this correspondence, ergodic measures are identified with extremal traces.

Let $\mathfrak{A}$ be a simple AF-algebra, and $\mathcal{T} \subset \mathfrak{A}$ a strongly maximal TAF subalgebra. Let $\mathcal{D}=\mathcal{T} \cap \mathcal{T}^{*}$ and $X=\mathcal{D}^{\wedge}$. $\mathcal{T}$ is said to be standard $\mathbb{Z}$-analytic if $\mathcal{T}$ can be written as a direct limit of direct sums of upper triangular matrix algebras with standard embeddings. If $X_{\max }\left(X_{\min }\right)$ denotes those points having no immediate successor (predecessor), then $X_{\max }\left(X_{\min }\right)$ is easily seen to be nonempty closed, nowhere dense in $X$. (A more general result, which applies to strongly maximal TAF algebras, is proved in [11, Proposition III. 5].) Furthermore, there is a homeomorphism $\varphi: X \backslash X_{\max } \rightarrow X \backslash X_{\min }$ which assigns to each $x$ in its domain the immediate successor of $X$. The orbit of $x \in X$ is

$$
\begin{aligned}
{[x] } & =\left\{\sigma_{v}(x): v \text { a matrix unit in } \mathfrak{A} \text { with } x \in \widehat{v v^{*}}\right\} \\
& =\left\{\varphi^{n}(x): n \in \mathbb{Z}, \quad x \in \operatorname{dom} \varphi^{n}\right\}
\end{aligned}
$$


Also, the half-orbit $\{y \in X: x \prec y\}$

$$
\begin{aligned}
& =\left\{\sigma_{v}(x): v \text { a matrix unit in } \mathcal{T} \text { with } x \in \widehat{v v^{*}}\right\} \\
& =\left\{\varphi^{n}(x): n \in \mathbb{N} \cup\{0\}, \quad x \in \operatorname{dom} \varphi^{n}\right\} .
\end{aligned}
$$

(See $[\mathbf{1 0}],[\mathbf{1 6}]$ for more facts about analyticity and $\mathbb{Z}$-analyticity.)

Proposition III.3.1. Suppose the simple AF algebra $\mathfrak{A}$ admits a tracial state and $\mathcal{T} \subseteq \mathfrak{A}$ is a standard $\mathbb{Z}$-analytic TAF subalgebra. Then there is an irreducible representation $\rho$ of $\mathfrak{A}$ such that $\rho(\mathcal{T})$ weakly dense in $\mathcal{B}\left(\mathcal{H}_{\rho}\right)$.

Proof. If $\mathfrak{A}$ admits a tracial state, it has an extremal tracial state; let $\mu$ be the corresponding invariant, ergodic probability measure on $X$. As $X_{\max }, \varphi^{-1}\left(X_{\max }\right), \ldots, \varphi^{-n}\left(X_{\max }\right)$ are disjoint with equal $\mu$-measure, each must have measure zero. Similarly, $X_{\min }$, $\varphi\left(X_{\min }\right), \ldots$ each has measure zero. Thus $\varphi$ may be regarded as an invertible measure-preserving transformation on the measure space $(X, \mu)$. The ergodicity of $\mu$ w.r.t. the maps $\sigma_{v}$ is equivalent to saying $\mu$ is ergodic w.r.t. $\varphi$, by earlier remarks.

Next, suppose $D \subseteq X$ is measurable and decreasing; i.e. $\varphi^{-1}(D) \subseteq$ $D$. Let $E=\bigcup_{n=0}^{\infty} \varphi^{n}(D)$. Then $\varphi^{-1}(E)=E=\varphi(E)$. By ergodicity, $\mu(E)=0$ or 1 . But $\mu\left(\left(\varphi^{n}(D)\right) \Delta D\right)=0$ so $\mu(D \Delta E)=0$; i.e. $\mu(D)=0$ or 1 . By Proposition II.2,

$$
\text { Lat } \tilde{\mathcal{T}}=\{0,1\} \text {, and } \tilde{\mathcal{T}}=\operatorname{Alg}\{0,1\}=\mathcal{B}\left(\mathcal{H}_{\rho}\right)
$$

While Theorem III.1.1 was motivated by Proposition 0.1 and uses only that $\pi$ is an irreducible representation of ambient UHF algebra, the next proposition, which applies to the larger class of $\mathbb{Z}$-analytic TAF algebras, uses the result of Proposition II.2.1 that $\rho$ is an Arveson represention.

Proposition III.3.2. Let $\mathcal{T}$ be a $\mathbb{Z}$-analytic TAF subalgebra of a simple AF algebra $\mathfrak{A}$, and $\rho$ an irreducible masa-preserving representation of $\mathfrak{A}$. Then $\mathcal{N}=$ Lat $\rho(\mathcal{T})$ has the order type of a subset 
of the integers.

Proof. Setting $X$ the Gelfand space of $\mathcal{T} \cap \mathcal{T}^{*}$, we may suppose $\rho=\rho_{\mu, \alpha}$ for some quasi-invariant probability measure $\mu$ on $X$ and 1-cocycle $\alpha$. By Proposition 2, $\mathcal{N}$ may be identified with the decreasing, measurable subsets of $X$. Suppose $E \subset X$ is such a set. With notation as above,

$$
E \supseteq \varphi^{-1}(E) \supseteq \cdots \supseteq \varphi^{-n}(E) \supseteq \cdots
$$

is a chain of decreasing sets, as is

$$
E \subseteq E \cup \varphi(E) \subseteq \cdots \subseteq \bigcup_{\imath=0}^{n} \varphi^{i}(E) \subseteq \ldots
$$

Either chain may stabilize at some point. In any case, if we can show these are the only decreasing sets, the Proposition will follow. Since $\mathcal{N}$ is a nest, any $F \in \mathcal{N}$ not listed above must lie between two elements in the list, and changing notation we may suppose that $\varphi^{-1}(E) \subset F \subset E$. Set $F_{1}=(E \backslash F) \cup \varphi^{-1}(E)$; clearly $F_{1}$ is decreasing. Now $\mu\left(F_{1} \backslash F\right)=\mu(E \backslash F)$, and $\mu\left(F \backslash F_{1}\right)=\mu\left(F \backslash \varphi^{-1}(E)\right)$. Thus, if both $\mu(E \backslash F)>0$ and $\mu\left(F \backslash \varphi^{-1}(E)\right)>0$, it follows that $F_{1}, F$ are two decreasing sets which are not comparable. This contradicts that $\mathcal{N}$ is a nest, so no such $F$ exists.

III.4. On the variety of attainable order types. Next we give a strongly maximal TAF algebra $\mathcal{T}$ which can be faithfully represented both as a weakly dense subalgebra of the Volterra nest algebra, and as a weakly dense subalgebra of a nest algebra $\operatorname{Alg} \mathcal{N}$, where $\mathcal{N}$ has the order type of the natural numbers.

Let $\mathcal{T}_{k}$ be the standard algebra of upper triangular $2^{2^{k}} \times 2^{2^{k}}$ matrices. Set $[n]=2^{2^{n}}$. We embed $\mathcal{T}_{k}$ in $\mathcal{T}_{k+1}$ by the identification,

$$
e_{i, j}^{(k)}=e_{i, j}^{(k+1)}+\sum_{r=1}^{[k]-1} e_{i([k]-1)+r+1, j([k]-1)+r+1}^{(k+1)}
$$

for $1 \leq i, j \leq[k]$. Then we can form the strongly maximal triangular algebra $\mathcal{T}=\lim \mathcal{T}_{k}$. (Notice that this is a limit of algebras of upper triangular $2^{2^{k}}$ square matrices.) We shall see that there are masa-preserving representations $\rho_{1}, \rho_{2}$ of $\mathcal{T}$ such that $\rho_{1}(\mathcal{T})$ is 
weakly dense in a nest algebra whose lattice is ordered as the natural numbers and $\rho_{2}(\mathcal{T})$ is weakly dense in the Volterra nest algebra.

It is clear that the enveloping $C^{*}$-algebra for $\mathcal{T}$ is the UHF algebra with supernatural number $2^{\infty}$ and that the maximal ideal space of the diagonal of $\mathcal{T}$ can be identified with

$$
X=\prod_{n=0}^{\infty}\{0,1\}_{n} .
$$

Now the element $x_{0}=(0,0, \ldots)$ of $X$ corresponds to the decreasing sequence $e_{1,1}^{(k)}, k=1,2,3, \ldots$ and its orbit is ordered as the positive integers. We obtain $\rho_{1}$ by equipping this orbit with the counting measure.

On the other hand, if we let $\mu$ be the product $\prod_{0}^{\infty} \mu_{n}$ of the probability measures

$$
\mu_{n}\{0\}=\mu\{1\}=1 / 2
$$

then this is an invariant ergodic measure on $X$. Moreover, since $\mathcal{T}$ is strongly maximal triangular, the $\mu$-equivalence classes of decreasing sets are totally ordered. It remains to show that the range of $\mu$ on the decreasing sets is dense in the unit interval.

Fix $k=2^{l}$ and $1 \leq r \leq 2^{k}$. Let $p_{0}=\sum_{i=1}^{r} e_{i, i}^{(k)}$ and let $p_{0}=\sum_{i=1}^{r} e_{i, i}^{(k)}$ and let

$$
\widehat{p_{n}}=\bigcup_{i \leq j} e_{i, j}^{\left(\widehat{\left.k_{n}\right)} p_{0} e_{j, i}^{\left(k_{n}\right)}\right.}
$$

where $k_{n}=2^{l+n}$. We take $\tau$ to be the faithful trace on the diagonal of $\mathcal{T}$ corresponding to integration by $\mu$ and observe that $\tau\left(p_{0}\right)=$ $r 2^{-k}$. By the embedding relationship one checks that

$$
\tau\left(p_{1}\right)=\frac{2^{k}+r\left(2^{k}-1\right)}{2^{2 k}} \leq \tau\left(p_{0}\right)+2^{-k}
$$

By the same token,

$$
\tau\left(p_{2}\right) \leq \tau\left(p_{1}\right)+2^{-2 k} \leq \tau\left(p_{0}\right)+2^{-k}+2^{-2 k}
$$

and in general,

$$
\tau\left(p_{0}\right) \leq \tau\left(p_{n}\right) \leq \tau\left(p_{0}\right)+\sum 2^{-r k} \leq \tau\left(p_{0}\right)+2^{1-k} .
$$

The union of the subsets $\widehat{p_{n}}$ of $X$ corresponding to $p_{n}$ is a decreasing set in $X$ which has measure in the range $\left[r 2^{-k},(r+2) 2^{-k}\right]$. Since $r$ and $k$ were arbitrary, the result follows. 


\section{Multiplicity of represented algebras.}

\section{IV.1. A masa-preserving representation with multiplicity} two nest. In this section we present an example of a strongly maximal TAF algebra $\mathcal{T}$ which admits a masa-preserving representation in a nest algebra in which the nest has uniform multiplicity two, and the representation of the ambient UHF algebra is weakly dense in $\mathcal{B}(\mathcal{H})$.

Let $\mathcal{T}_{k}$ be the $2^{k}$-square upper triangular matrices. We shall suppose for convenience that the matrix units of $\mathcal{T}_{k}$ are indexed by sequences of zeroes and ones of length $k$. Embed $\mathcal{T}_{k}$ in $\mathcal{T}_{k+1}$ by means of the identification

$$
\begin{aligned}
e_{\left(i_{1}, \ldots, i_{k}\right),\left(j_{1}, \ldots, j_{k}\right)}^{(k)}= & e_{\left(i_{1}, \ldots, l_{k-1}, 0, i_{k}\right),\left(j_{1}, \ldots, j_{k-1}, 0, j_{k}\right)}^{(k+1)} \\
& +e_{\left(i_{1}, \ldots,,_{k-1}, 1, i_{k}\right),\left(j_{1}, \ldots, j_{k-1}, 1, j_{k}\right)}^{(k+1)} .
\end{aligned}
$$

Now the maximal ideal space, $X$, of the diagonal of $\mathcal{T}=\lim \mathcal{T}_{k}$ can be identified with the set of sequences $x=\left(x_{1}, x_{2}, \ldots ; x_{\omega}\right)$ where $x_{a}$ are each in $\{0,1\}$. The $\mathrm{S}-\mathrm{V}$ equivalence classes are the sets of those $x$ having common tail in the infinite segment (but possibly differing entries at the $\omega$ position). The ordering on the equivalence classes is lexicographic. As with the refinement algebra, we put a measure on the maximal ideal space of the diagonal corresponding to the product measure

$$
\mu=\prod_{n=1}^{\infty} \mu_{n} \times \mu_{\omega}
$$

where each of the measures has weight $1 / 2$ on both 0 and 1 . Now the $\mathrm{S}-\mathrm{V}$ representation arising from this measure can be shown to give a continuous nest of uniform multiplicity two. This can be shown directly, but perhaps the clearest way to see it is by introducing the mapping $\phi$ from $X$ to $[0,1]$ by

$$
\left(x_{1}, x_{2}, \ldots ; x_{\omega}\right) \mapsto x_{\omega} / 2+\sum_{n=1}^{\infty} x_{n} / 2^{n+1} .
$$

This map is absolutely continuous from $\mu$ to Lebesgue measure on the unit interval and one readily checks that the partial homeomorphisms $\hat{e}_{i, j}^{(k)}$ on $X$ correspond to the maps of $f$ to

$$
\chi_{\left[k(\imath), k(i)+1 / 2^{k}\right]}(t) f(t+k(j)-k(i))
$$


where the endpoints $k(i)$ are given by

$$
k(i)=\frac{[(i-1) / 2]}{2^{k}}+\left(\frac{i-1}{2}-\left[\frac{i-1}{2}\right]\right) .
$$

Then it is easily seen that the weakly closed algebra generated by these operators is the nest algebra whose nest is the projections $\chi_{[0, t]}+\chi_{\left[\frac{1}{2}, \frac{1}{2}+t\right]}$ for $0 \leq t \leq \frac{1}{2}$. Furthermore, from the ergodicity of Lebesgue measure under dyadic translations that $\mu$ is ergodic, and hence by Theorem II.1 the representation of the ambient UHF algebra is irreducible.

\section{IV.2. The failure of representations to preserve triangular-} ity for nest algebras. If $\rho$ is an irreducible representation $\mathfrak{A} \rightarrow$ $\mathcal{B}\left(\mathcal{H}_{\rho}\right)$ and $\mathcal{T} \subset \mathfrak{A}$ a TAF subalgebra, we have seen that $\tilde{\mathcal{T}}-\rho \overline{(\mathcal{T})}$ wk can fail to be triangular, even if $\rho$ is masa-preserving. Indeed, III.3.1 shows that $\tilde{\mathcal{T}}$ can be $\mathcal{B}\left(\mathcal{H}_{\rho}\right)$, while the previous example shows $\tilde{\mathcal{T}}$ can be the nest algebra of a nest of uniform multiplicity two. That $\tilde{\mathcal{T}}$ generally fails to be triangular in $\mathcal{B}\left(\mathcal{H}_{\rho}\right)$ is hardly surprising, as weak closure does not preserve triangularity. However if $\mathcal{T}$ is a nest algebra in the AF algebra $\mathfrak{A}$ such that Lat $\mathcal{T}$ generates the diagonal of $\mathcal{T}$ in $\mathfrak{A}$, and $\rho: \mathfrak{A} \rightarrow \mathcal{B}\left(\mathcal{H}_{\rho}\right)$ is an irreducible, masa-preserving representation, we have seen that $\tilde{\mathcal{T}}$ is necessarily triangular (Corollary III.1.4). One question that remains is this: if we take $\mathcal{T}$ to be a nest algebra, but drop the assumption that Lat $\mathcal{T}$ generate the diagonal in $\mathfrak{A}$, is it still true that $\widetilde{\mathcal{T}}$ must be triangular?

In this example we present a triangular nest subalgebra $\mathcal{T}$ of a UHF algebra $\mathfrak{A}$, and an irreducible masa-preserving representation $\rho$ of $\mathfrak{A}$ such that the weak closure $\tilde{\mathcal{T}}=\rho \overline{(\mathcal{T})}^{\text {wk }}$ is a nest algebra, but is not a triangular subalgebra of $\mathcal{B}\left(\mathcal{H}_{\rho}\right)$; specifically, $\tilde{\mathcal{T}} \cap \tilde{\mathcal{T}}^{*}$ is a non-commutative von Neumann algebra which contains a masa in $\mathcal{B}(\mathcal{H})$.

We recall the construction of the TUHF algebra $\mathcal{T}_{(\alpha)}$ from $\left[8\right.$, Theorem 4.5] and [9, Theorem 2.24]. Let $\mathfrak{A}_{n}=M_{2^{n}},\left\{e_{i j}^{(n)}\right\}_{1 \leq i, j \leq 2^{n}}$ be matrix units for $\mathfrak{A}_{n}$, and write diagonal matrix units $e_{i i}^{(n)}$ as $e_{i}^{(n)}$ for convenience. For $N \in \mathbb{N}$, set $Q(N)$ the permutation matrix in $M_{2 N}$ such that

$$
\begin{gathered}
Q(N) \operatorname{diag}\left(a_{1}^{(1)}, a_{2}^{(1)}, a_{1}^{(2)}, a_{2}^{(2)}, \ldots a_{1}^{(N)}, a_{2}^{(N)}\right) Q(N)^{*} \\
\operatorname{diag}\left(a_{1}^{(1)}, a_{1}^{(2)}, \ldots a_{1}^{(N)}, a_{2}^{(1)}, a_{2}^{(2)}, \ldots, a_{2}^{(N)}\right)
\end{gathered}
$$


(Here $\operatorname{diag}\left(b_{1}, \ldots, b_{\ell}\right)$ denote the diagonal matrix in $M_{\ell}$.) For each $n$ and $1 \leq m<2^{n}$, let

$$
R(n, m)=\left[\begin{array}{c|c}
I_{2 m} & 0 \\
\hline 0 & Q\left(2^{n}-m\right)
\end{array}\right]
$$

Observe that $\operatorname{Ad} R(n, m)$ maps upper triangular matrices in $\mathfrak{A}_{n}$ to upper triangular matrices in $\mathfrak{A}_{n+1}$. For $\alpha \in(0,1)$, let $\alpha=\sum_{n=1}^{\infty} \frac{k_{n}}{2^{n}}$ be the nonterminating binary expansion. Set $M_{n}=\sum_{\ell=1}^{n} 2^{n-i} k_{i}, \theta_{n}=$ Ad $R\left(n, M_{n}\right) \circ \nu_{n}, \mathfrak{A}=\varliminf_{\longrightarrow}\left(\mathfrak{A}_{n}, \theta_{n}\right)$ (a realization of UHF $\left.2^{\infty}\right), \mathcal{D}=$ $\longleftrightarrow\left(\mathcal{D}_{n}, \theta_{n}\right)$, where $\mathcal{D}_{n}$ is the diagonal subalgebra of $\mathfrak{A}_{n}$, and $\mathcal{T}_{(\alpha)}=$ $\varliminf_{\longrightarrow}\left(\mathcal{T}_{n}, \theta_{n}\right)$, where $\mathcal{T}_{n}$ is the upper triangular subalgebras of $\mathfrak{A}_{n}$. Let $X$ be the Gelfand spectrum of $\mathcal{D}$. Let $\mathcal{N}=\{p \in \mathcal{D}: p=$ $\left.\sum_{i=1}^{k} e_{i}^{(n)}, k=1, \ldots, M_{n}, n=1,2, \ldots\right\} \cup\{0,1\}$.

In [9] it is shown that $\mathcal{N}=\operatorname{Lat}\left(\mathcal{T}_{(\alpha)}\right)$ and $\mathcal{T}_{(\alpha)}=\operatorname{Alg}(\mathcal{N})$. Let $Z \subseteq X, Z=\cup\{\hat{p}: p \in \mathcal{N}, p<1\}$. Since (as proved in [8]) $\sup \{\operatorname{tr}(p): p \in \mathcal{N}, p<1\}=\alpha$, we have $\mu(Z)=\alpha$, where $\alpha$ is the probability measure on $X$ corresponding to the unique normalized trace on $\mathfrak{A}$. Set $Y=X \backslash Z$. If $x \in X$, let $f(n, x)$ denote the unique integer $j$ such that $x \in \hat{e}_{j}^{(n)}$.

LEMmA IV.2.1. If $x \in X$, limsup $\left[\frac{f(n, x)}{2^{n}}\right] \geq \alpha$, then $\frac{f(n, x)}{2^{n}} \geq$ $\alpha$ for all $n$.

Proof. Since $\frac{M_{n}}{2^{n}}$ is the truncation of the binary expansion of $\alpha, \frac{M_{n}}{2^{n}}<\alpha \leq \frac{M_{n}+1}{2^{n}}$. Thus, if for some $n, \frac{f(n, x)}{2^{n}}<\alpha$, then $f(n, x) \leq M_{n}$. By definition of the embedding $\theta_{n}$,

$$
\theta_{n}\left(e_{j}^{(n)}\right)=e_{2 j-1}^{(n+1)}+e_{2 j}^{(n+1)}, \quad j \leq M_{n} .
$$

As $x \in \hat{e}_{2 j-1}^{(n+1)}$ or $x \in \hat{e}_{2 j}^{(n+1)}, f(n+1, x) \leq 2 f(n, x)$; so that for $m>n, f(m, x) \leq 2^{m-n} f(n, x)$. It follows

$$
\frac{f(m, x)}{2^{m}} \leq \frac{f(n, x)}{2^{n}} \leq \frac{M_{n}}{2^{n}}
$$


So

$$
\limsup _{m} \frac{f(m, x)}{2^{m}} \leq \frac{M_{n}}{2^{n}}<\alpha
$$

Corollary IV.2.2. $Z=\left\{x \in X: \lim \sup \frac{f(n, x)}{2^{n}}<\alpha\right\}$.

Proof. If $x \in Z$, then $x \in \hat{p}$ for some $p \in \mathcal{N}, p<1$. Say $p=$ $\sum_{i=1}^{j} e_{i}^{(n)} ;$ as observed earlier $j \leq M_{n}$, so $f(n, x) \leq j \leq M_{n}$. It follows from the Lemma that $\lim \sup \frac{f(n, x)}{2^{n}}<\alpha$.

If on the other hand $\limsup \frac{f(n, x)}{2^{n}}<\alpha$, then by the Lemma $\frac{f(n, x)}{2^{n}}<\alpha$ for some $n$. Hence $f(n, x) \leq M_{n}$, and $x \in \hat{p}, p=$ $\sum_{i=1}^{f(n, x)} e_{i}^{(n)} \in \mathcal{N}$. So $x \in Z$.

Since $\mathcal{T}$ is strongly maximal and triangular, the ordering (defined prior to Theorem II.1) on orbits of $X$ is a total order on each orbit. By [10] a projection $p \in \mathcal{D}$ belongs to $\mathcal{N}$ iff $\hat{p}$ is a clopen, decreasing set. If $p_{n}=\sum_{i=1}^{M_{n}} e_{i}^{(n)}$, then the sets $\left\{\hat{p}_{n}: n=1,2, \ldots\right\}$ form an increasing sequence of decreasing, clopen sets, hence their union (which is $Z$ ) is decreasing and open.

Lemma IV.2.3. $Z$ is decreasing, open and dense in $X$.

Proof. It remains to show $Z$ is dense. To show every nonempty open set intersects $Z$, it is enough to show that if $e_{j}^{(n)}$ is a matrix unit, $\hat{e}_{j}^{(n)} \cap Z \neq \emptyset$. Now if $j \leq M_{n}, \hat{e}_{j}^{(n)} \subseteq Z$, so we may assume $j>M_{n}$, say $j=M_{n}+j_{0}$.

Now

$$
\begin{aligned}
\theta_{n}\left(e_{M_{n}+j_{0}}^{(n)}\right) & =e_{M_{n+1}+j_{0}-k_{n+1}}^{(n+1)}+e_{M_{n+1}+j_{0}-k_{n+1}+L_{n}}^{(n+1)} \\
\left(L_{n}=2^{n}-M_{n}\right) & \geq e_{M_{n+1}+j_{0}-k_{n+1}}^{(n+1)}
\end{aligned}
$$


Since the dyadic expansion $\alpha=\sum_{i=1}^{\infty} \frac{k_{i}}{2^{i}}$ is non terminating there is an $m>n$ with $k_{n+1}+\cdots+k_{m}=j_{0}$. It follows that $\theta_{m-1} \circ \cdots \circ$ $\theta_{n}\left(e_{j}^{(n)}\right) \geq e_{M_{m}}^{(m)}$, and since $\hat{e}_{M_{m}}^{(m)} \subset Z$, we are done.

Corollary IV.2.4. $Y$ is closed, increasing, nowhere dense. Also. $\mu(Y)=1-\alpha$.

Any strongly maximal TUHF algebra $\mathcal{T}=\overline{\cup \mathcal{T}_{n}}$, where $\mathcal{T}_{n}$ is isomorphic to the upper triangular matrices in some finite factor, has the property that there is a unique maximal element in the spectrum of the diagonal; specifically, there is exactly one orbit having a maximal element, and the remaining orbits have no maximal element. This applies, in particular, to $\mathcal{T}_{(\alpha)}$. Let $y_{\max }$ be the unique maximal element in $X$ :

$$
\left\{y_{\max }\right\}=\bigcap_{n=1}^{\infty} \hat{e}_{2^{n}}^{(n)} \subset Y .
$$

LEMmA IV.2.5. Every element of $Y \backslash\left\{y_{\max }\right\}$ has an immediate successor in the induced ordering on the orbits of $Y$.

Proof. If $y \in Y, y \neq y_{\max }$, then $y \in e_{j}^{(n)}$ with $j<2^{n}$, for sufficiently large $n$. Since by Lemma IV.2.1, Cor. IV.2.2, $j=f(n, y)>$ $M_{n}$ for all $n, \theta_{n}\left(e_{j j+1}^{(n)}\right)=e_{j_{0} j_{0}+1}^{(n+1)}+e_{j_{1} j_{1}+1}^{\left(n_{1}\right)}$ for some $j_{0}, j_{1}$, it follows that $\varphi(y)=\bigcap_{m=n}^{\infty} \hat{e}_{f(m, y)+1}^{(m)}$ is nonempty, and hence intersects in a unique point, denoted $\varphi(y)$. Observe

$$
(y, \varphi(y)) \in \hat{e}_{f(m, y), f(m, y)+1}^{(m)}, \quad m \geq n .
$$

If $y^{\prime} \in[y]$ satisfies $y \prec y^{\prime}$, then for some $m \geq n,\left(y, y^{\prime}\right) \in \hat{e}_{f(m, y), f\left(m, y^{\prime}\right)}^{(m)}$ with $f\left(m, y^{\prime}\right)>f(m, y)$. Thus, $\varphi(y) \prec y^{\prime}$.

Corollary IV.2.6. The map $\varphi$ is a homeomorphism on its domain.

Proof. Let $y \in \operatorname{dom}(\varphi)$ and $j$ as above. As $\varphi\left(y^{\prime}\right)=\sigma_{v}\left(y^{\prime}\right)$ for $y^{\prime} \in \hat{e}_{j}^{(n)}, v=e_{j j+1}^{(n)}$, i.e., the restriction of $\varphi$ to the clopen set $\hat{e}_{j}^{(n)}$ coincides with the partial homeomorphism $\sigma_{v} . \varphi$ is a homeomorphism on its domain. 
Note that if $\left\{y_{\min }\right\}=Y \backslash \varphi\left(Y \backslash\left\{y_{\max }\right\}\right)$ then $\varphi: Y \backslash\left\{y_{\max }\right\} \rightarrow$ $Y \backslash\left\{y_{\min }\right\}$ is a homeomorphism. Here $y_{\min }$ is the unique minimal element of $Y$; $y_{\min }$ is not minimal in $X$. If $y \in Y$, the intersection of the orbit $[y]$ in $X$ with $Y,[y]_{Y}=\left\{\varphi^{n}(y): n \in \mathbb{Z}, y \in \operatorname{dom} \varphi^{n}\right\}$ where $\varphi^{2}=\varphi \circ \varphi$ with appropriate domain, $\varphi^{3}=\varphi^{2} \circ \varphi$, etc., and $\varphi^{-1}$ is the inverse map, $\varphi^{-2}=\varphi^{-1} \circ \varphi^{-1}$, etc. Since the measure $\mu$ is invariant under the partial homeomorphisms $\sigma_{v}, v$ a matrix unit, it is invariant under $\varphi$. Furthermore, since the orbits $\left[y_{\max }\right]_{Y},\left[y_{\min }\right]_{Y}$ are countable and hence null, we can regard $\varphi$ as an invertible measurepreserving transformation of $\left(Y, \mu^{\prime}\right), \mu^{\prime}=\left.\frac{1}{1-\alpha} \mu\right|_{Y}$. For if $\nu$ were another invariant probability measure on $Y$, then $E \mapsto \mu(E \cap Z)+$ $(1+\alpha) \nu(E \cap Y)$ would be an invariant probability measure on $X$, and hence by uniqueness of the trace, equal to $\mu$. It follows $\nu=\mu^{\prime}$. Thus $\mu^{\prime}$ is ergodic w.r.t. $\varphi$.

LEMmA IV.2.7. Let $E \subset X$ be any decreasing, $\mu$-measurable set containing $Z$. Then $E=Z$ or $E=X$ (up to measure zero).

Proof. Let $F=E \cap Y$. Then $F$ is a decreasing subset of $Y$, and regarding $\varphi$ as measure-preserving transformation of $Y, \varphi^{-1}(F) \subseteq$ $F$, with $\mu\left(\varphi^{-1}(F)\right)=\mu(F)$. Let $F_{0}=\bigcup_{n=1}^{\infty} \varphi^{n}(F)$. Then $\mu(F)=$ $\mu\left(\varphi^{n}(F)\right)$, so $\nu(F)=\mu\left(F_{0}\right) . F_{0}$ is invariant in $Y$. By ergodicity of $\varphi, F_{0}=\emptyset$ or $F_{0}=Y$ a.e. Thus, $F=\emptyset$ or $F=Y$ a.e. Since $E=F \cup Z$, the result follows.

CoROLlaRY IV.2.8. $\tilde{\mathcal{T}}_{(\alpha)}=\rho_{\mu}{\overline{\left(\mathcal{T}_{(\alpha)}\right)}}^{\text {wk }}$ is a nest algebra which is not triangular in $\mathcal{B}\left(\mathcal{H}_{\rho}\right)$.

Proof. By [3, Cor. 15.12] and Proposition II.2, $\widetilde{\mathcal{T}}_{(\alpha)}$ is a nest algebra. To show that $\tilde{\mathcal{T}}_{(\alpha)}$ is not triangular (in the sense of Kadison and Singer) in $\mathcal{B}\left(\mathcal{H}_{\rho}\right)$, it is enough to observe that $\operatorname{Lat}\left(\tilde{\mathcal{T}}_{(\alpha)}\right)$ is not a maximal nest in $\mathcal{H}_{\rho}$. As the successor of $L^{2}(Z)$ is $L^{2}(X)=\mathcal{H}_{\rho}$ in the nest, and $\operatorname{dim}\left(\mathcal{H}_{\rho} \ominus L^{2}(Z)\right)=\operatorname{dim} L^{2}(Y)>1$, Lat $\widetilde{\mathcal{T}}_{(\alpha)}$ is not a maximal nest in $\mathcal{H}_{\rho}$, and so the nest algebra $\widetilde{\mathcal{T}}_{\alpha}$ is not triangular. 


\section{IV.3. A non masa-preserving representation of the refine-} ment algebra with a multiplicy two nest. The TAF algebra $\mathcal{T}$ of Example IV.1 has the property that Lat $\mathcal{T}$ does not generate the SV masa $\mathcal{T} \cap \mathcal{T}^{*}$ in the ambient UHF algebra $\mathfrak{A}$. In fact the commutant of Lat $\mathcal{T}$ in $\mathfrak{A}$ is $M_{2}$, so Lat $\mathcal{T}$ is a multiplicity two nest in $\mathfrak{A}$ (see $[\mathbf{9}, 2.27])$. In this light, the existence of a multiplicity two representation is not surprising. On the other hand, if $\mathcal{S}$ is any strongly maximal TAF algebra such that $C^{*}($ Lat $\mathcal{S})=\mathcal{S} \cap \mathcal{S}^{*}$, then any $*$-extendable irreducible representation $\rho$ of $\mathcal{S}$ such that $\rho\left(\mathcal{S} \cap \mathcal{S}^{*}\right)$ is weakly dense in in a masa in $\mathcal{B}\left(\mathcal{H}_{\rho}\right)$ has the property that Lat $\rho(\mathcal{S})$ is a multiplicity one nest in $\mathcal{B}\left(\mathcal{H}_{\rho}\right)$.

Let $\mathcal{T}$ be the refinement algebra (I.1). From the above discussion, since Lat $\mathcal{T}$ generates the diagonal masa of $\mathcal{T}$, there is no irreducible masa-preserving representation $\rho$ such that Lat $\rho(\mathcal{T})$ is a multiplicity two nest. Yet one can ask if there is an irreducible, $*$-extendable representation $\rho$ such that Lat $\rho(\mathcal{T})$ is a multiplicity two nest in $\mathcal{B}\left(\mathcal{H}_{\rho}\right)$. The following example answers this affirmatively.

Set $u_{0}=\left[\begin{array}{ll}1 & 0 \\ 0 & 1\end{array}\right], u_{1}=\left[\begin{array}{ll}0 & 1 \\ 1 & 0\end{array}\right], u_{2}=\left[\begin{array}{cc}1 & 0 \\ 0 & -1\end{array}\right]$, and $u_{3}=\left[\begin{array}{cc}0 & -1 \\ 1 & 0\end{array}\right]$. The elements $\left\{ \pm u_{i}: 0 \leq i \leq 3\right\}$ form a subgroup of $U(2, \mathbb{R})$, the $2 \times 2$ real unitary matrices, satisfying the relations

$$
u_{1} u_{2}=u_{3}, \quad u_{2} u_{3}=-u_{1}, \quad \text { and } \quad u_{3} u_{1}=-u_{2} .
$$

Hence $u_{i}^{*} u_{j} u_{i}= \pm u_{j}$ for $0 \leq i, j \leq 3$.

For all $k \geq 1$ and $1 \leq i \leq 2^{k}-1$, inductively define unitaries $u_{i}^{(k)}$ by the relations

$$
u_{1}^{(k)}=u_{k} \bmod 4
$$

and

$$
u_{i}^{(k-1)}=u_{2 i-1}^{(k)} u_{2 i}^{(k)}=u_{2 i}^{(k)} u_{2 i+1}^{(k)} .
$$

Note that this implies

$$
u_{2 i+1}^{(k)}=u_{i}^{(k-1) *} u_{2 i-1}^{(k)} u_{i}^{(k-1)}
$$

and hence that for $i$ odd, $u_{i}^{(k)}= \pm u_{1}^{(k)}$.

Let $e_{i j}^{(k)}$ be the matrix units of the usual representation of the refinement algebra $\mathcal{T}$ which is dense in the Volterra algebra; that is, we write $e_{i, j}^{(k)}$ instead of $\rho\left(e_{i, j}^{(k)}\right)$ as in Example I.1. Let

$$
\tilde{e}_{i, i}^{(k)}=e_{i, i} \otimes u_{0}
$$


and

$$
\tilde{e}_{i, i+1}^{(k)}=e_{i, i+1}^{(k)} \otimes u_{i}^{(k)}
$$

The defining relation for the $u_{i}^{(k)}$ insures that the $\tilde{e}_{i, i+1}^{(k)}$ generate a representation, $\tilde{\mathcal{T}}$, of the refinement algebra. Set

$$
\begin{aligned}
S_{k} & =\sum_{i} e_{2 i-1,2 i}^{(k)}, \quad \text { and } \\
S_{k}^{+} & =\sum\left\{e_{2 i-1,2 i}^{(k)}: \text { over } i \text { such that } u_{2 i-1}^{(k)}=u_{[k]}\right\} \\
S_{k}^{-} & =\sum\left\{e_{2 i-1,2 i}^{(k)}: \text { over } i \text { such that } u_{2 i-1}^{(k)}=-u_{[k]}\right\}
\end{aligned}
$$

with $[k]=k \bmod 4$. Now $S_{k}^{+} \otimes u_{[k]}, S_{k}^{-} \otimes u_{[k]}$ belong to $\tilde{\mathcal{T}}$, as does their sum, $S_{k} \otimes u_{[k]}$. As $\left\{S_{k}\right\}_{k=1}^{\infty}$ converges weakly to $\frac{1}{2} I,\left\{S_{k} \otimes u_{[k]}\right\}$ converges weakly to $\frac{1}{2} I \otimes u_{[k]}$ as $k$ runs through the subsequence with $[k]$ constant. Because $\left\{u_{[k]}: k=0,1,2,3\right\}$ generate $M_{2}(\mathbb{C})$, the weak closure of $\tilde{\mathcal{T}}$ contains $I \otimes M_{2}(\mathbb{C})$. Of course it also follows that the diagonal of the weak closure of $\widetilde{\mathcal{T}}$ contains a masa, and so the result follows.

Acknowledgements. We would like to thank Alan Hopenwasser and Warren Wogen for helpful discussions.

\section{REFERENCES}

[1] W. B. Arveson, Operator algebras and invariant subspaces, Ann. Math., 1002 (1974), 433-532.

[2] R. L. Baker, Triangular UHF algebras, J. Funct. Anal., 91 (1990), 182212.

[3] K. R. Davidson, Nest algebras, Pitman Research Notes in Mathematics Series 191 John Wiley \& Sons New York 1988.

[4] A. Hopenwasser and S. C. Power, Classification of limits of triangular matrix algebras, Proc. Edinburg Math. Soc., 36 (1992), 107-121.

[5] R. V. Kadison and I. M. Singer, Triangular operator algebras, Amer. J. Math., 82 (1960), 227-259.

[6] P. S. Muhly and B. Solel, Subalgebras of groupoid $C^{*}$-algebras, J. Reine Angew. Math., 402 (1989), 41-75.

[7] G. K. Pederson, $C^{*}$-Algebras and their automorphism groups, London Math. Soc. Monograph No. 14, Academic Press, 1979. 
[8] J. R. Peters, Y. T. Poon, and B. H. Wagner, Triangular AF algebras, J. Operator Theory, 23 (1990), 81-114.

[9] J. R. Peters and B. H. Wagner, Triangular AF algebras and nest subalgebras of UHF algebras, J. Operator Theory, 25 (1991), 79-13.

[10] J. R. Peters, Y. T. Poon, and B. H. Wagner, Analytic TAF algebras, Canad. J. Math., 45 (1993), 1009-1031, Correction 46 (1994), 395-396.

[11] J. R. Peters and W. R. Wogen, Reflexive subalgebras of AF algebras, J. Funct. Anal., 122 (1994), 1-24.

[12] J. Renault, A Groupoid Approach to $C^{*}$-Algebras, Springer Lect. Notes in Math., 793 (1980).

[13] B. Solel, Irreducible triangular algebras, Mem. Amer. Math. Soc., No. 290 (1984).

[14] S. Stratila and D. Voiculescu, Representations of AF Algebras and of the Group $U(\infty)$, Springer Lect. Notes in Math., 486 (1975).

[15] M. Thelwall, Maximal triangular subalgebras of AF algebras, J. Operator Theory, 25 (1991), 163-176

[16] B. Ventura, Strongly maximal triangular UHF Algebras, Internat. J. Math., 2 (1991), 567-598.

Received June 5, 1992 and in revised form July 22, 1993.

UNIVERSITY OF NEBRASKA

LINCOLN, NE 68588

AND

IOWA StATE UNIVERSITY

AMES, IA 50011 



\title{
PACIFIC JOURNAL OF MATHEMATICS
}

Founded by

\author{
E. F. BeCKenBACH (1906-1982) F. Wolf (1904-1989)
}

EDITORS

Sun-Yung A. Chang

(Managing Editor)

University of California

Los Angeles, CA 90024-1555

pacific@math.ucla.edu

F. Michael Christ

University of California

Los Angeles, CA 90024-1555

christ@math.ucla.edu

ThOMAS ENRIGHT

University of California

San Diego, La Jolla, CA 92093

tenright@ucsd.edu
Nicholas ERCOLANI

University of Arizona

Tucson, AZ 85721

ercolani@math.arizona.edu

R. FINN

Stanford University

Stanford, CA 94305

finn@gauss.stanford.edu

VAUGhan F. R. Jones

University of California

Berkeley, CA 94720

vfr@math.berkeley.edu

SteVEn KeRcKHofF

Stanford University

Stanford, CA 94305

spk@gauss.stanford.edu
MARTIN SCHARLEMANN

University of California

Santa Barbara, CA 93106

mgscharl@math.ucsb.edu

\section{GANG TIAN}

Courant Institute

New York University

New York, NY 10012-1110

tiang@taotao.cims.nyu.edu

V. S. VARADARAJAN

University of California

Los Angeles, CA 90024-1555

vsv@math.ucla.edu

\section{SUPPORTING INSTITUTIONS}

UNIVERSITY OF ARIZONA

UNIVERSITY OF BRITISH COLUMBIA

CALIFORNIA INSTITUTE OF TECHNOLOGY

UNIVERSITY OF CALIFORNIA

UNIVERSITY OF MONTANA

UNIVERSITY OF NEVADA, RENO

NEW MEXICO STATE UNIVERSITY

OREGON STATE UNIVERSITY

\author{
UNIVERSITY OF OREGON \\ UNIVERSITY OF SOUTHERN CALIFORNIA \\ STANFORD UNIVERSITY \\ UNIVERSITY OF HAWAII \\ UNIVERSITY OF UTAH \\ WASHINGTON STATE UNIVERSITY \\ UNIVERSITY OF WASHINGTON
}

The Supporting Institutions listed above contribute to the cost of publication of this Journal, but they are not owners or publishers and have no responsibility for its content or policies.

Mathematical papers intended for publication in the Pacific Journal of Mathematics should be in typed form or offset-reproduced (not dittoed), double spaced with large margins. Please do not use built up fractions in the text of the manuscript. However, you may use them in the displayed equations. Underline Greek letters in red, German in green, and script in blue. The first paragraph must be capable of being used separately as a synopsis of the entire paper. In particular it should contain no bibliographic references. Please propose a heading for the odd numbered pages of less than 35 characters. Manuscripts, in triplicate, may be sent to any one of the editors. Please classify according to the 1991 Mathematics Subject Classification scheme which can be found in the December index volumes of Mathematical Reviews. Supply name and address of the author to whom proofs should be sent. All other communications should be addressed to the managing editor, or Julie Honig, University of California, Los Angeles, California 90024-1555.

There are page-charges associated with articles appearing in the Pacific Journal of Mathematics. These charges are expected to be paid by the author's University, Government Agency or Company. If the author or authors do not have access to such Institutional support these charges are waived. Single authors will receive 75 free reprints; joint authors will receive a total of 100 free reprints. Additional copies may be obtained at cost in multiples of 50 .

The Pacific Journal of Mathematics (ISSN 0030-8730) is published monthly except for July and August. Regular subscription rate: $\$ 215.00$ a year (10 issues). Special rate: $\$ 108.00$ a year to individual members of supporting institutions.

Subscriptions, orders for numbers issued in the last three calendar years, and changes of address should be sent to Pacific Journal of Mathematics, P.O. Box 4163, Berkeley, CA 94704-0163, U.S.A. Old back numbers obtainable from Kraus Periodicals Co., Route 100, Millwood, NY 10546.

The Pacific Journal of Mathematics at University of California, c/o Department of Mathematics, 981 Evans Hall, Berkeley, CA 94720 (ISSN 0030-8730) is published monthly except for July and August. Second-class postage paid at Berkeley, CA 94704, and additional mailing offices. POSTMASTER: send address changes to Pacific Journal of Mathematics, P.O. Box 4163, Berkeley, CA 94704-0163.

PUBLISHED BY PACIFIC JOURNAL OF MATHEMATICS at University of California,

Berkeley, CA 94720, A NON-PROFIT CORPORATION

This publication was typeset using $\mathcal{A} \mathcal{M} \mathcal{S}$ - IATEX,

the American Mathematical Society's TEX macro system.

Copyright (C)1994 by Pacific Journal of Mathematics 


\section{PACIFIC JOURNAL OF MATHEMATICS}

Volume $167 \quad$ No. $1 \quad$ January 1995

Local reproducing kernels on wedge-like domains with type 2 edges

Al Boggess and AlexANDER NAGEL

Discriminants of involutions on Henselian division algebras

MaUrice Chacron, H. DHERTE, JEAN-Pierre Tignol,

ADRIAN R. WADSWORTH and V. I. YANCHEVSKIĬ

Essential tori obtained by surgery on a knot

MARIO EUdAVE-MuÑOZ

Non-compact totally peripheral 3-manifolds

LUKE HARRIS and PETER SCOTT

Some representations of TAF algebras

JOHN LINDSAY ORR and JUSTIN PETERS

A non-Haken hyperbolic 3-manifold covered by a surface bundle

ALAN W. REID

The nonhomogeneous minimal surface equation involving a measure 